Boletín de la Sociedad Geológica Mexicana

Volumen 61, NÚM. 3, 2009, P. 287-303

\title{
Geocronología y distribución espacial del vulcanismo en el Campo Volcánico de San Luis Potosí
}

\author{
Margarito Tristán-González ${ }^{1, *}$, Alfredo Aguillón-Robles ${ }^{1}$, José Rafael Barboza-Gudiñoㅇ, \\ José Ramón Torres-Hernández ${ }^{1}$, Hervé Bellon², Rubén López-Doncel ${ }^{1}$, \\ Rodolfo Rodríguez-Ríos ${ }^{1,3}$, Guillermo Labarthe-Hernández ${ }^{1}$ \\ ${ }^{1}$ Instituto de Geología/DES Ingeniería, Universidad Autónoma de San Luis Potosí, Av. Dr. Manuel Nava \#5, \\ Zona Universitaria, C.P. 78240, San Luis Potosí, S.L.P., México. \\ ${ }^{2}$ UMR 6538, Domaines Océaniques, IUEM, Université de Bretagne Occidentale, \\ 6, Av. Le Gorgeu, BP 809, F-29285 Brest Cedex, Francia. \\ ${ }^{3}$ Facultad de Ingeniería/DES Ingeniería, Área Ciencia de la Tierra, Universidad Autónoma de San Luis Potosí, \\ Av. Dr. Manuel Nava \#8, Zona Universitaria, C.P. 78240, San Luis Potosí, S.L.P., México. \\ *mtristan@uaslp.mx
}

Resumen

El Campo Volcánico de San Luis Potosí, se localiza en la porción sur-oriental de la Mesa Central y está formado principalmente por una secuencia de lavas y flujos piroclásticos con edades que van desde el Eoceno medio hasta el Cuaternario. En general, el Campo Volcánico de San Luis Potosí tuvo cinco etapas principales de vulcanismo. La primera etapa estuvo restringida al Eoceno medio y consistió en la emisión de lavas andesíticas. La segunda etapa fue la más voluminosa y sucedió en el período entre 32 y 28 Ma. En ésta segunda etapa predominó la actividad efusiva que formó cadenas de domos exógenos con composiciones que varían desde dacitas hasta riolitas con contenido alto de sílice. La formación de estos domos fue precedida por erupciones piroclásticas de volumen menor que las lavas. La tercera es una etapa intermitente de vulcanismo félsico que sucedió entre los 28-25 Ma y originó erupciones piroclásticas de la riolita Panalillo. La expulsión de la riolita Panalillo al parecer fue a través de fallas normales, formadas durante la etapa en que la extensión fue más intensa, lo que sucedió en el lapso entre los 28-26 Ma. La expulsión de la riolita Panalillo en algunos complejos estuvo acompañada de basaltos por lo que se interpreta como actividad bimodal. La cuarta etapa de vulcanismo ocurrió entre los 2321 Ma con erupciones fisurales esporádicas de basaltos a través de fallas, lo cual ocurrió principalmente en la parte sur y sur-poniente de la Sierra de San Miguelito; la última etapa, se asocia a un vulcanismo intraplaca que se traslapó sobre algunos de los complejos volcánicos que conforman el Campo Volcánico de San Luis Potosí durante el Cuaternario, cuyos centros eruptivos generaron rocas de composición basanítica.

El vulcanismo del Campo Volcánico de San Luis Potosí está compuesto por una diversidad magmática y multiepisódica, ampliamente asociada a los eventos de la tectónica extensiva de la porción central de México originada principalmente durante el Oligoceno.

Los complejos eruptivos, es decir los complejos volcánicos principales que conforman el Campo Volcánico de San Luis Potosí, presentan ciertas diferencias entre sí, pero son correlacionables por sus características petrológicas, las cuales van de acuerdo a sus edades isotópicas. Para este trabajo se seleccionaron seis complejos volcánicos: Ahualulco, Pinos, Villa Hidalgo, La Repartición, Sierra San Miguelito y Santa María. Se determinaron edades isotópicas K-Ar de la mayoría de sus unidades, las cuales están de acuerdo con su posición estratigráfica.

Palabras clave: Edades K-Ar, vulcanismo del Cenozoico, Campo Volcánico de San Luis Potosí, vulcanismo en la Mesa Central.

\section{Abstract}

The San Luis Potosi volcanic field, is located in the southeastern of the Mesa Central, mainly formed by a sequence of lavas and pyroclastic flows with ages between the Middle Eocene until the Quaternary. Generally in the San Luis Potosi Volcanic Field there are five mainly volcanic stages; the first one restringed to the middle Eocene with the emission of andesitic lavas; the second stage is the 
more voluminous and occurred between 32 to $28 \mathrm{Ma}$, dominated by effusive activity that was formed by exogenous domes where the composition are from dacitic to rhyolitic lavas with high silica contain. The formation of these domes were preceded by pyroclastic eruption in minor volume that the lavas flows; the third one stage forming by intermittent felsic volcanism occurred between 28 to 25 Ma formed by pyroclastic eruption of the Panalillo rhyolite associated to normal faults of the maximum extension tectonic event occurred between 28-26 Ma; in some volcanic centers were accompanying by basaltic lava flows showing a bimodal volcanism; the fourth one stage occurred between 23-21 Ma represented by fissural eruptions of basalts extruded through the faults, that mainly occurred in the southern and southwestern of the Sierra de San Miguelito. The last stage was an intraplate volcanism that has overlied to some volcanic centers in the San Luis Potosi Volcanic Field during the Quaternary period; these volcanic centers extruded basanitic composition magmas.

The San Luis Potosi volcanic field volcanism is associated to magmatic diversity and multiepisodic, associated to extensive tectonic events of the central-north of Mexico mainly originated between the Oligocene times.

The eruptive complexes, in the same way the main volcanic complexes that forming the volcanic field, show some difference but are correlationated by petrologic characteristics and by isotopic ages. In this work, we are selected six volcanic complexes: Ahualulco, Pinos, Villa Hidalgo, La Repartición, Sierra San Miguelito and Santa María. We obtained K-Ar isotopic ages in the most unities, and they are concordant with the stratigraphy position.

Key words: K-Ar ages, Cenozoic volcanism, San Luis Potosí volcanic field, Mesa Central volcanism.

\section{Introducción}

El Campo Volcánico de San Luis Potosí (CVSLP, Figura 1), cubre un área aproximada de $40,000 \mathrm{~km}^{2}$ y se ha definido como tal, con base en los trabajos de cartografía geológica desarrollados en la porción centro-sur del Estado de San Luis Potosí por los investigadores del Instituto de Geología de la UASLP y otras instituciones, reconociendo un paquete de unidades volcánicas que se formó desde el Eoceno al Cuaternario (Labarthe-Hernández et al., 1982). Los estudios realizados se encaminaron a determinar la estratigrafía y estructura de las rocas volcánicas del CVSLP con base en descripciones de campo, petrografía y geoquímica de trabajos publicados previamente y complementados con 56 nuevos fechamientos K-Ar de roca entera. La cartografía se realizó sistemáticamente partiendo de la Sierra San Miguelito y extrapolándose a los otros complejos volcánicos que conforman el CVSLP (Labarthe-Hernández et al., 1982; Labarthe-Hernández y Jiménez-López, 1992; 1993; 1994; Labarthe-Hernández et al., 1995; 1999; Aguillón-Robles, 1992; Barboza-Gudiño et al., 2001; 2002). Otro aspecto importante, son los trabajos que se realizaron para interpretar los eventos tectónicos asociados al emplazamiento del vulcanismo regional en el CVSLP (Tristán-González, 1986; 1987, Tristán-González y Torres-Hernández, 1992; Nieto-Samaniego et al., 1996; Nieto-Samaniego et al., 2005; Tristán-González et al., 2008).

El vulcanismo del CVSLP inició en el Eoceno medio con la emisión de derrames de lava andesítica depositadas sobre sedimentos lacustres del Paleoceno tardío al Eoceno temprano-medio (Labarthe-Hernández et al., 1982). Posteriormente, en el Oligoceno, se depositó un paquete de lavas y productos piroclásticos de composición intermedia a riolítica. El período donde se emitió el volumen mayor de rocas volcánicas félsicas en el CVSLP, fue entre los 32 y 26 Ma. Entre los 22 y $20 \mathrm{Ma}$ el vulcanismo fue muy restringido, con carácter bimodal (se emitieron basaltos y riolitas casi de manera contemporánea) y finalizó en etapas intermitentes con lavas basaníticas del Plio-Cuaternario.

Aunque el carácter químico de las lavas e ignimbritas del CVSLP es predominantemente calcialcalino (LabartheHernández et al., 1982), y por lo tanto asociado a un régimen de convergencia, el evento de extensión tectónica que formó la Provincia de Cuencas y Sierras; parece haber impuesto su sello en el vulcanismo, dado que están ligados vulcanismo y extensión en la Mesa Central de México (Aranda-Gómez et al., 2000; Torres-Hernández et al., 2001; Aguirre-Díaz y Labarthe-Hernández, 2003). Stewart y Roldán-Quintana (1994), consideran que la deformación y el vulcanismo asociado a la subducción pudieron continuar hasta el Mioceno temprano. De cualquier manera, el carácter predominantemente calcialcalino de las rocas, el tiempo restringido de su emplazamiento y la bimodalidad química de las rocas de la SMO son típicas de magmatismo de arco continental (McDowell y Kaizer, 1977). Sin embargo, reconocemos que de acuerdo con Wark, et al. (1990); Wark, (1991) que el cambio de énfasis en el carácter del vulcanismo (de calcialcalino a subalcalino y alcalino) después de los $30 \mathrm{Ma}$ en la parte norte de la SMO y tardíamente en su parte sur-occidental (en el CVSLP) entre los 22 y $20 \mathrm{Ma}$ (Mioceno temprano) parece indicar el paso de un régimen dominado por procesos de subducción, a un episodio de extensión menor. Aranda-Gómez et al. (2000) sitúan un periodo de extensión en toda la región sur de la provincia tectónica de Cuencas y Sierras entre 24 y $20 \mathrm{Ma}$.

Este trabajo, tiene el propósito de dar a conocer edades isotópicas nuevas, obtenidas por el método $\mathrm{K}-\mathrm{Ar}$ en roca entera o fase mineral, y hacer una comparación entre 


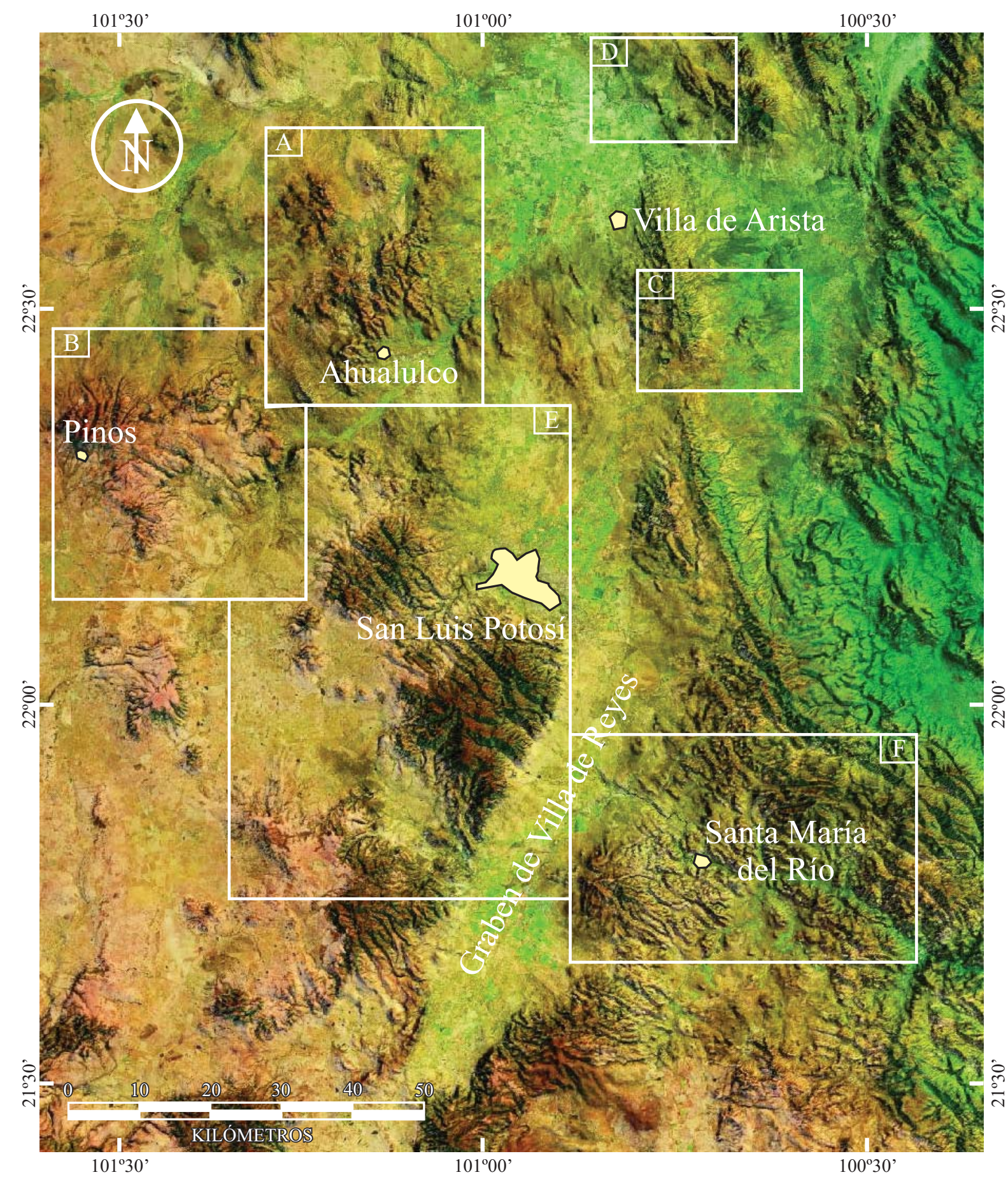

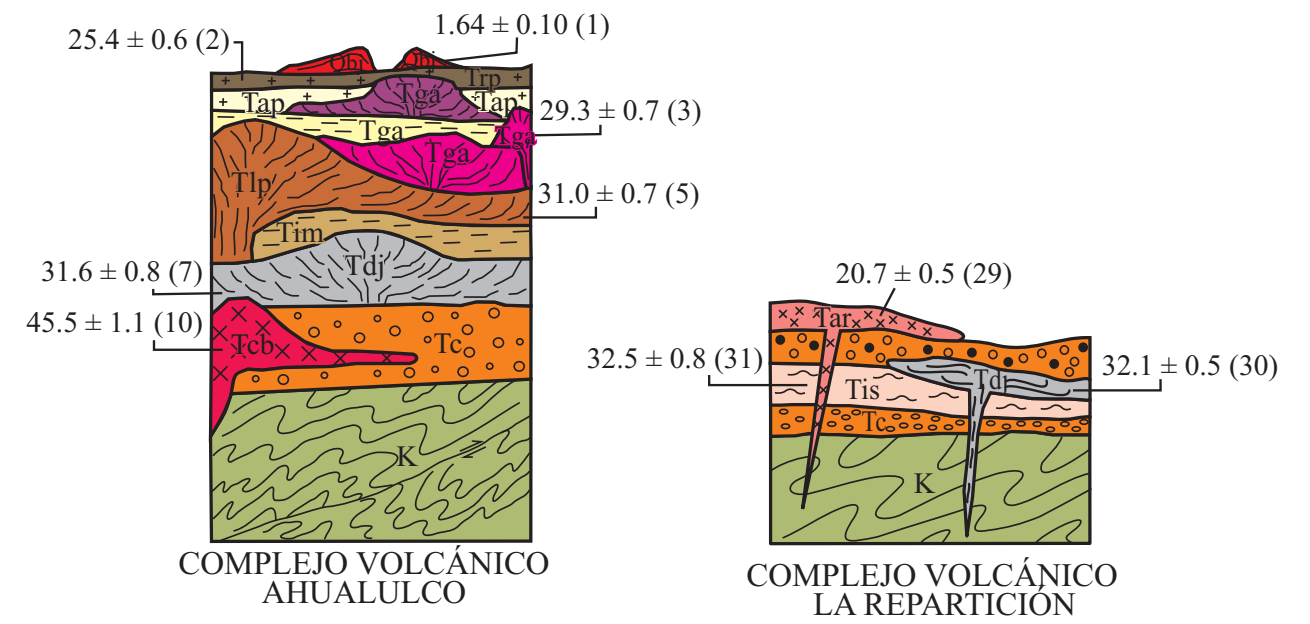

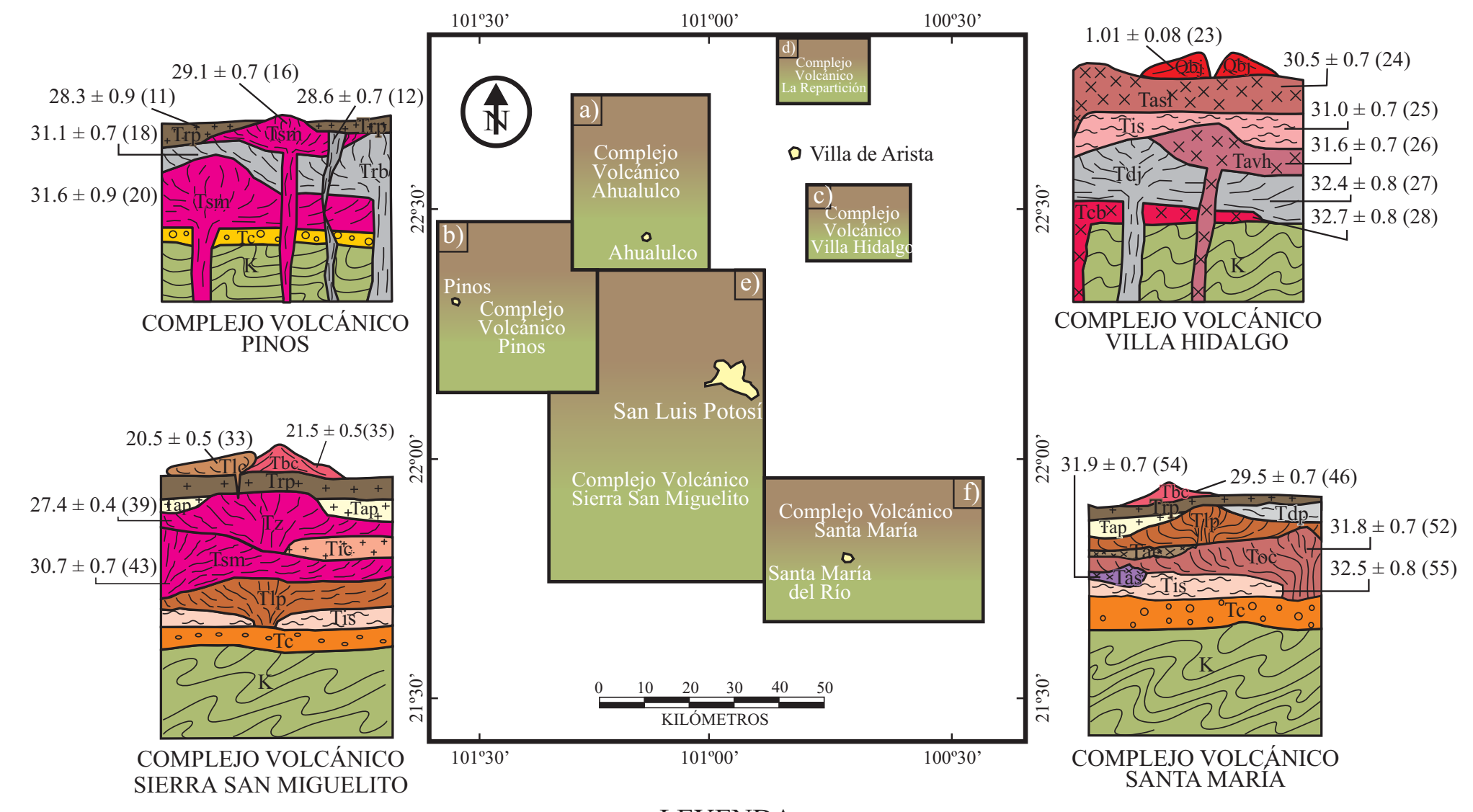

\begin{tabular}{ll} 
2bj & Basalto Las Joyas \\
\hline$T l c$ & Traquita Los Castillo \\
\hline $\mathrm{Tar}$ & Andesita La Repartición \\
$\mathrm{Tbc}$ & Basalto Cabras \\
\hline $\mathrm{Tr} p$ & Ignimbrita Panalillo Superior \\
\hline $\mathrm{Tap}$ & Ignimbrita Panalillo Inferior \\
\hline $\mathrm{Tz}$ & Riolita El Zapote
\end{tabular}

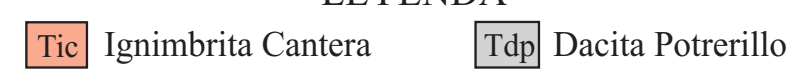

Tasl Andesita San Lorenzo

Tsm Riolita San Miguelito Tim Ignimbrita Membrillo

\begin{tabular}{lll}
\hline Tcu Riolita Cuervos & Trb Riodacita Barbecho
\end{tabular}

Tth Tobo Hienera

Tavh Andesita Villa Hidalgo

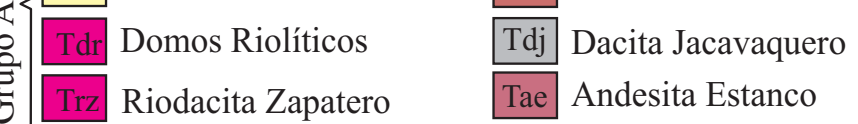

Toc Traquita Ojo Caliente

Tis Ignimbrita Santa María

Tas Andesita Salitrera

$\mathrm{Tcb}$ Andesita Casita Blanca

Tc Formación Cenicera

$\mathrm{K}$ Cretácico marino indiferenciado 
estas edades nuevas y las edades publicadas de rocas provenientes del CVSLP (Labarthe-Hernández et al., 1982; Aguillón-Robles et al., 1994; Nieto-Samaniego et al., 1996; Aranda-Gómez et al., 2007). También pretende hacer una separación de las localidades que de acuerdo a su semejanza en la estratigrafía, geoquímica, geocronología y control tectónico, permita dividir el CVSLP en complejos volcánicos; los cuales se incluyen en este trabajo, y son nombrados como: Ahualulco, Pinos, Villa Hidalgo, La Repartición, Sierra San Miguelito y Santa María (Figura 1). Las edades que aquí se reportan fueron realizadas en el laboratorio de geocronología de la Universidad de Bretaña Occidental mediante la cooperación científica entre la UBO y la UASLP.

\section{Método analítico}

Las edades isotópicas por el método K-Ar, fueron obtenidas de muestras procedentes de lavas e ignimbritas, preparadas en roca entera y en fase mineral. Después de quebrar y cribar la roca a una fracción del tamaño entre 0.3 a $0.15 \mu \mathrm{m}$, fueron lavadas con agua destilada para luego proceder con el método analítico: (i) una parte se reduce a polvo en un mortero de ágata para realizar el análisis de $\mathrm{K}$ por absorción atómica después de un ataque químico con ácido fluorhídrico, y (ii) los granos de 0.3 a $0.15 \mu \mathrm{m}$ fueron utilizados para el análisis isotópico de Ar. La extracción del Argón se realizó en alto vacío y por calentamiento de la muestra por inducción en un crisol de molibdeno. Los gases extraídos fueron purificados en dos filtros de Ti y finalmente se purificó el Ar utilizando dos filtros de Al-Zr SAES. La composición isotópica de Ar y la concentración de ${ }^{40} \mathrm{Ar}$ radiogénico se midieron usando un espectrómetro de masas de acero inoxidable con una geometría de $180^{\circ}$, equipado con un amplificador Keithley 642. El método de dilución isotópica se aplicó usando un trazador de ${ }^{38} \mathrm{Ar}$ que fue implantado como ion en una pequeña hoja de aluminio. Todo el método original es descrito por Bellon et al. (1981).

Los fechamientos, con los parámetros respectivos, se muestran en la Tabla 1 y fueron calculados usando la constante recomendada por Steiger y Jäger (1977), y la incertidumbre del error de $\pm 1 \sigma$ se calculó con la ecuación de Cox y Dalrymple (1967), para muestras de roca más antigua a 5 Ma, y con la ecuación de Mahood y Drake (1982) para las muestras más jóvenes a $1 \mathrm{Ma}$.

\section{Geología de los complejos volcánicos}

La tectónica extensiva, generada desde el Eoceno, que afectó la porción occidental de la Mesa Central (LabartheHernández et al., 1982; Tristán-González, 1986; NietoSamaniego et al., 1997; Aranda-Gómez y McDowell, 1998; Nieto-Samaniego et al., 1999; Aranda-Gómez et al.,
2000; Nieto-Samaniego et al., 2005), fue un mecanismo importante para el emplazamiento de lavas y depósitos piroclásticos que fueron emitidos por diferentes complejos eruptivos del CVSLP. Estos complejos presentan variaciones geoquímicas importantes, caracterizándose por ser contemporáneos y co-magmáticos. A partir del Oligoceno en los diferentes complejos volcánicos fueron emplazados derrames de lavas que formaron cadenas de domos exógenos y depósitos piroclásticos de composiciones que varían desde dacita o andesita rica en potasio hasta riolita, o derrames de lava andesítica a basáltica, asociadas a los diferentes patrones tectónicos de fallas o fisuras del Paleoceno (Tristán-González et al., 2008).

La separación de los complejos volcánicos del CVSLP se realizó por correlación de unidades volcánicas de acuerdo a su semejanza litológica-estructural, posición estratigráfica, análisis químicos y edades isotópicas. Así se tiene el Complejo Volcánico Ahualulco (CVA) localizado al norte del CVSLP (Figura 1a; LabartheHernández y Tristán-González, 1981; Aguillón-Robles y Tristán-González, 1981; Labarthe-Hernández et al., 1982; Labarthe-Hernández et al., 1995). Complejo Volcánico Pinos (CVP) se ubica en la porción poniente del CVSLP (Figura 1b; Aguillón-Robles, 1983; Aguillón-Robles et al., 1994a; Aguillón-Robles et al., 1996; Rodríguez-Ríos, 1997; Rocha-Echavarría et al., 2006; Aranda-Gómez et al., 2007). Complejo Volcánico Villa Hidalgo (CVVH) y La Repartición (CVR) ubicados al nororiente del CVSLP (Figura 1c y d; Barboza-Gudiño et al., 2001; BarbozaGudiño et al, 2002). Complejo Volcánico Sierra San Miguelito (CVSSM), que conforma la mayor parte del CVSLP (Figura 1e; Labarthe-Hernández et al., 1982; Labarthe-Hernández y Jiménez-López, 1992, 1993, 1994; Torres-Hernández et al., 2006). Complejo Volcánico Santa María (CVSM) localizado al occidente del Campo Volcánico del Río Santa María (Labarthe-Hernández et al., 1984; Tristán-González, 1987; Aguillón-Robles et al., 2004).

\subsection{Complejo Volcánico Ahualulco}

El Complejo Volcánico de Ahualulco (Figura 1A y 2), se localiza en la porción norte del CVSLP (LabartheHernández y Tristán-González, 1981; Aguillón-Robles y Tristán-González, 1981; Labarthe-Hernández et al., 1982; Labarthe-Hernández et al., 1995). El basamento pre-volcánico, son sedimentos flysch de la Formación Caracol (Tardy et al., 1975; Carrillo-Bravo, 1982; Silva-Romo, 1993), formado de una secuencia de capas de arenisca feldespática y lutita físil del Cretácico superior. Sobre la Formación Caracol se depositaron en cuencas aisladas sedimentos continentales compuestos por secuencias de grava, limo y arcilla, pertenecientes a la Formación Cenicera del Paleoceno-Eoceno (Labarthe-Hernández et al., 1982). Sobre los sedimentos continentales se derramaron lavas de 
Tabla 1. Edades K-Ar, de muestras colectadas y analizadas para el CVSLP.

\begin{tabular}{|c|c|c|c|c|c|c|c|c|c|c|c|c|}
\hline SG & Muestra & Tipo de roca & $\begin{array}{l}\text { Unidad } \\
\text { geológica }\end{array}$ & $\begin{array}{c}\text { Edad } \\
\pm 1 \sigma\end{array}$ & $\begin{array}{l}\text { Longitud } \\
\text { (W) }\end{array}$ & $\begin{array}{l}\text { Latitud } \\
\text { (N) }\end{array}$ & Fracción & $\begin{array}{c}{ }^{40} \mathrm{Ar}_{\mathrm{R}} \\
\left(10^{-7} \mathrm{~cm}^{3} / \mathrm{g}\right)\end{array}$ & $\begin{array}{c}\% \\
{ }^{40} \mathbf{A r}_{\mathrm{R}}\end{array}$ & $\begin{array}{c}\mathrm{K}_{2} \mathrm{O} \\
(\% \mathrm{wt})\end{array}$ & $\begin{array}{c}\text { Peso } \\
\text { (g) }\end{array}$ & $\begin{array}{l}\text { Ref.* } \\
\text { Lab }\end{array}$ \\
\hline \multicolumn{13}{|c|}{ Complejo Volcánico Ahualulco } \\
\hline 1 & SLP 01-25 & basalto & Qbj & $1.50 \pm 0.8$ & 101.0694 & 22.5894 & $\mathrm{RE}$ & 1.09 & 18.1 & 2.06 & 0.8045 & 6171 \\
\hline 2 & SLP 01-24 & Ign riolítica & Trp & $25.4 \pm 0.6$ & 101.0694 & 22.4814 & $\mathrm{RE}$ & 49.3 & 81.7 & 2.25 & 0.6013 & 6170 \\
\hline 3 & SLP 01-28 & dacita & $\operatorname{Tn}$ & $29.3 \pm 0.7$ & 101.2303 & 22.4169 & $\mathrm{f}$ & 46.7 & 63.8 & 4.90 & 0.5022 & 5965 \\
\hline 4 & SLP 01-32 & riolita & Tga & $31.0 \pm 0.7$ & 101.2405 & 22.3747 & $\mathrm{RE}$ & 54.6 & 82.7 & 5.41 & 0.6085 & 6184 \\
\hline 5 & SLP 01-22 & dacita & Tlp & $31.0 \pm 0.7$ & 101.1225 & 22.5203 & $\mathrm{~m}$ & 47.7 & 68.7 & 5.22 & 0.3500 & 5978 \\
\hline 6 & SLP 01-29 & dacita & Tga & $31.2 \pm 0.7$ & 101.1894 & 22.4105 & $\mathrm{RE}$ & 59.5 & 86.6 & 5.86 & 0.6040 & 6183 \\
\hline 7 & SLP 01-21 & dacita & Tdj & $31.6 \pm 0.8$ & 101.1708 & 22.5080 & $\mathrm{RE}$ & 47.5 & 75.4 & 4.70 & 0.6046 & 6169 \\
\hline 8 & SLP 01-30 & dacita & Tip & $32.0 \pm 0.8$ & 101.1369 & 22.5455 & $\mathrm{RE}$ & 63.4 & 79.6 & 6.09 & 0.6040 & 6182 \\
\hline 9 & SLP 01-33 & andesita & $\mathrm{Tcb}$ & $44.4 \pm 1.0$ & 101.2244 & 22.5311 & $\mathrm{RE}$ & 28.5 & 82.4 & 1.79 & 06068 & 6240 \\
\hline 10 & SLP 01-31 & andesita & Tcb & $45.5 \pm 1.1$ & 101.1711 & 22.5911 & $\mathrm{RE}$ & 26.6 & 72.5 & 1.79 & 0.6133 & 6241 \\
\hline \multicolumn{13}{|c|}{ Complejo Volcánico Pinos } \\
\hline 11 & SLP 01-09 & ign riolítica & Trp & $28.3 \pm 0.9$ & 101.3344 & 22.2100 & $\mathrm{RE}$ & 56.1 & 54.4 & 6.10 & 0.610 & 6148 \\
\hline 12 & SLP 01-08 & riodacita & $\operatorname{Trb}$ & $28.6 \pm 0.7$ & 101.4869 & 22.3430 & $\mathrm{f}$ & 45.1 & 83.2 & 4.85 & 0.210 & 5961 \\
\hline 13 & SLP 01-07 & riolita & Tsm & $28.7 \pm 0.8$ & 101.2252 & 22.2252 & $\mathrm{RE}$ & 62.4 & 72.1 & 6.70 & 0.610 & 6142 \\
\hline 14 & SLP 01-10 & ign riolítica & $\operatorname{Trp}$ & $29.0 \pm 0.9$ & 101.3522 & 22.4088 & $\mathrm{RE}$ & 51.4 & 59.2 & 5.46 & 0.600 & 6149 \\
\hline 15 & SLP 01-11 & ign riolítica & Trp & $29.0 \pm 0.9$ & 101.4692 & 22.2853 & $\mathrm{RE}$ & 49.4 & 63.1 & 5.23 & 0.600 & 6156 \\
\hline 16 & SLP 01-12 & riolita & Tsm & $29.1 \pm 0.7$ & 101.3177 & 22.3736 & $\mathrm{RE}$ & 48.1 & 63.8 & 5.08 & 0.610 & 6157 \\
\hline 17 & SLP 01-04 & riolita & Tsm & $30.3 \pm 0.9$ & 101.5005 & 22.3708 & $\mathrm{RE}$ & 60.0 & 80.5 & 6.10 & 0.600 & 6147 \\
\hline 18 & SLP 01-02 & riolita & Tsm & $31.1 \pm 0.7$ & 101.5611 & 22.3216 & $\mathrm{f}$ & 52.8 & 87.7 & 5.23 & 0.5035 & 6244 \\
\hline 19 & SLP 01-03 & riodacita & $\operatorname{Trb}$ & $31.1 \pm 0.7$ & 101.4908 & 22.2280 & bi & 70.7 & 80.4 & 6.89 & 0.100 & 5970 \\
\hline 20 & SLP 01-05 & riolita & Tsm & $31.6 \pm 0.9$ & 101.0694 & 22.5894 & $\mathrm{RE}$ & 56.2 & 87.1 & 5.47 & 0.600 & 6150 \\
\hline 21 & SLP 01-01 & riolita & Tsm & $32.0 \pm 0.7$ & 101.5347 & 22.3527 & $\mathrm{~m}$ & 57.6 & 89.9 & 5.54 & 0.6006 & 6238 \\
\hline \multicolumn{13}{|c|}{ Complejo Volcánico Villa Hidalgo } \\
\hline 22 & TG 49 & basalto & Qbj & $0.59 \pm 0.06$ & 100.6297 & 22.2761 & $\mathrm{RE}$ & 0.41 & 10.0 & 2.15 & 1.0056 & 6479 \\
\hline 23 & TG 43 & basalto & Qbj & $1.01 \pm 0.10$ & 100.6594 & 22.5572 & $\mathrm{RE}$ & 0.75 & 12.0 & 2.30 & 1.0035 & 6470 \\
\hline 24 & SLP 01-35 & andesita & Tasl & $30.5 \pm 0.7$ & 100.6750 & 22.4472 & $\mathrm{RE}$ & 27.0 & 63.2 & 2.72 & 0.6044 & 6232 \\
\hline 25 & VH-153 & Ign riolítica & Tis & $31.0 \pm 0.7$ & 100.6419 & 22.5000 & $\mathrm{RE}$ & 53.9 & 81.7 & 5.35 & 0.6056 & 6940 \\
\hline 26 & SLP 03-01 & andesita & Tavh & $31.6 \pm 0.7$ & 100.8230 & 22.4463 & $\mathrm{RE}$ & 18.8 & 72.0 & 1.83 & 1.0010 & 6451 \\
\hline 27 & VH-183 & dacita & Tdj & $32.4 \pm 0.8$ & 100.6799 & 22.4697 & $\mathrm{RE}$ & 29.5 & 85.3 & 2.80 & 0.4000 & 7002 \\
\hline 28 & $135 \mathrm{VH}$ & andesita & $\mathrm{Tcb}$ & $32.7 \pm 0.8$ & 100.6515 & 22.4733 & $\mathrm{RE}$ & 18.8 & 60.0 & 1.77 & 0.6014 & 6930 \\
\hline \multicolumn{13}{|c|}{ Complejo Volcánico la Repartición } \\
\hline 29 & SLP 01-13 & andesita & Tar & $20.7 \pm 0.5$ & 100.8366 & 22.8588 & $\mathrm{RE}$ & 11.9 & 58.4 & 1.78 & 0.6200 & 6158 \\
\hline 30 & SLP 00-20 & dacita & Tdj & $32.1 \pm 0.5$ & 100.7972 & 22.7950 & $\mathrm{RE}$ & 30.0 & 85.2 & 2.88 & 0.2507 & 5549 \\
\hline 31 & SLP 01-15 & ign riolítica & Tis & $32.5 \pm 0.8$ & 100.7977 & 22.7972 & $\mathrm{RE}$ & 57.8 & 59.2 & 5.47 & 0.6000 & 6159 \\
\hline \multicolumn{13}{|c|}{ Complejo Volcánico Sierra San Miguelito } \\
\hline 32 & SLP 99-01 & traquita & Tlc & $20.3 \pm 0.5$ & 101.4954 & 21.9048 & $\mathrm{RE}$ & 41.1 & 93.6 & 6.26 & 0.4057 & 5127 \\
\hline 33 & SLP 08-03 & traquita & Tlc & $20.5 \pm 0.5$ & 102.0691 & 21.3788 & $\mathrm{RE}$ & 41.3 & 70.2 & 6.22 & 0.5010 & 6464 \\
\hline 34 & SLP 99-04 & traquita & Tlc & $20.9 \pm 0.5$ & 101.5080 & 22.2755 & $\mathrm{RE}$ & 45.6 & 87.6 & 6.72 & 0.5055 & 5134 \\
\hline 35 & SLP 99-02 & basalto & $\mathrm{Tbc}$ & $21.5 \pm 0.5$ & 101.4815 & 22.3675 & $\mathrm{RE}$ & 8.5 & 74.1 & 1.22 & 0.5074 & 5126 \\
\hline 36 & SLP 00-06 & riolita & Tsm & $21.1 \pm 0.3$ & 101.2191 & 22.0063 & $\mathrm{RE}$ & 37.1 & 90.9 & 5.43 & 0.2521 & 5551 \\
\hline 37 & SLP 00-18 & ign riolítica & Tap & $25.7 \pm 0.4$ & 101.1058 & 22.0963 & $\mathrm{f}$ & 40.1 & 74.7 & 4.81 & 0.2518 & 5527 \\
\hline 38 & SLP 00-14 & basalto & Tbp & $26.9 \pm 0.4$ & 101.1558 & 22.0863 & $\mathrm{RE}$ & 26.3 & 79.4 & 3.02 & 0.5032 & 5523 \\
\hline 39 & SLP 00-19 & riolita & $\mathrm{Tz}$ & $27.4 \pm 0.4$ & 101.3877 & 21.8588 & $\mathrm{f}$ & 39.5 & 90.4 & 5.16 & 0.3041 & 5580 \\
\hline 40 & SLP 00-02 & basalto & Tbp & $28.0 \pm 0.6$ & 101.1522 & 22.1427 & $\mathrm{RE}$ & 26.5 & 55.5 & 2.91 & 0.5079 & 5496 \\
\hline 41 & SLP 00-03 & ign riolítica & Trp & $28.9 \pm 0.5$ & 101.1491 & 22.1438 & $\mathrm{RE}$ & 49.1 & 67.3 & 5.24 & 0.3099 & 5570 \\
\hline 42 & SLP 00-17 & riolita & Tsm & $30.4 \pm 0.5$ & 101.2552 & 22.1069 & $\mathrm{f}$ & 42.1 & 85.5 & 4.26 & 0.4013 & 5637 \\
\hline 43 & SLP 01-38 & riolita & Tsm & $30.7 \pm 0.7$ & 101.2550 & 22.1047 & $\mathrm{RE}$ & 34.1 & 89.6 & 3.63 & 0.6024 & 6187 \\
\hline 44 & SLP 01-36 & riolita & Tsm & $31.3 \pm 0.7$ & 101.0866 & 22.0083 & $\mathrm{RE}$ & 52.9 & 81.1 & 5.20 & 0.6015 & 6185 \\
\hline 45 & SLP 01-37 & riolita & Tsm & $32.7 \pm 1.0$ & 101.0750 & 22.0369 & $\mathrm{RE}$ & 32.5 & 39.5 & 3.05 & 0.6091 & 6189 \\
\hline
\end{tabular}

SG: Sitio geológico, localización de las muestras analizadas (K-Ar), en cada complejo volcánico. Unidad geológica: estratigrafía reportada por LabartheHernández et al., (1982). $\pm 1 \sigma$; calculado siguiendo la ecuación de Cox y Dalrymple (1967), en muestras de roca más antigua a 5 Ma, y la ecuación dada por Mahood y Drake (1982) para muestras más jóvenes a 1 Ma. Fracción; RE, roca entera; m, matriz; f, feldespato; bi, biotita. \% p.: por ciento en peso de potasio. Ref. Lab.: Base de datos en el Laboratorio de Geocronología de la Universidad de Bretaña Occidental. 
Tabla 1. (Cont.) Edades K-Ar, de muestras colectadas y analizadas para el CVSLP.

\begin{tabular}{|c|c|c|c|c|c|c|c|c|c|c|c|c|}
\hline SG & Muestra & Tipo de roca & $\begin{array}{l}\text { Unidad } \\
\text { geológica }\end{array}$ & $\begin{array}{c}\text { Edad } \\
\pm 1 \sigma\end{array}$ & $\begin{array}{l}\text { Longitud } \\
\text { (W) }\end{array}$ & $\begin{array}{l}\text { Latitud } \\
\text { (N) }\end{array}$ & Fracción & $\begin{array}{c}{ }^{40} \mathrm{Ar}_{\mathrm{R}} \\
\left(10^{-7} \mathrm{~cm}^{3} / \mathrm{g}\right)\end{array}$ & $\begin{array}{c}\% \\
{ }^{40} \mathbf{A r}_{\mathrm{R}}\end{array}$ & $\begin{array}{c}\mathbf{K}_{2} \mathrm{O} \\
(\% \mathbf{p})\end{array}$ & $\begin{array}{c}\text { Peso } \\
\text { (g) }\end{array}$ & $\begin{array}{l}\text { Ref.* } \\
\text { Lab }\end{array}$ \\
\hline \multicolumn{13}{|c|}{ Complejo Volcánico Río Santa María } \\
\hline 46 & TG 52 & andesita & & $29.5 \pm 0.7$ & 100.8861 & 21.7733 & $\mathrm{RE}$ & 12.8 & 77.9 & 1.34 & 1.0124 & 6497 \\
\hline 47 & SLP 01-44 & ign riolítica & Tis & $31.3 \pm 0.7$ & 100.7344 & 21.8077 & $\mathrm{RE}$ & 52 & 81.3 & 5.10 & 0.6014 & 6287 \\
\hline 48 & SLP $07-03$ & sienita & Tipv & $31.5 \pm 0.7$ & 100.5416 & 21.8222 & RE & 52.4 & 85.1 & 5.12 & 0.5330 & 6461 \\
\hline 49 & JAG 27-03 & traquita & Toc & $31.6 \pm 0.7$ & 100.7805 & 21.8875 & RE & 66.1 & 56.3 & 6.44 & 0.5030 & 6457 \\
\hline 50 & SLP 99-03 & andesita & Tae & $31.6 \pm 0.7$ & 100.4333 & 21.9752 & $\mathrm{RE}$ & 31.8 & 94.5 & 3.10 & 0.4004 & 5186 \\
\hline 51 & SLP 99-05 & ign riolítica & Tis & $31.7 \pm 0.5$ & 100.4811 & 21.9711 & $\mathrm{f}$ & 53.9 & 73.2 & 5.23 & 0.4115 & 5285 \\
\hline 52 & JAG 03-03 & traquita & Toc & $31.8 \pm 0.7$ & 100.4812 & 21.9712 & $\mathrm{RE}$ & 58.4 & 84.4 & 5.65 & 0.5027 & 6452 \\
\hline 53 & JAG 21-03 & traquita & Toc & $31.8 \pm 0.7$ & 100.7816 & 21.7841 & RE & 41.7 & 74.3 & 4.04 & 0.5043 & 6456 \\
\hline 54 & SLP 03-02 & andesita & Tas & $31.9 \pm 0.7$ & 100.6371 & 21.9634 & $\mathrm{RE}$ & 25.2 & 71.3 & 2.42 & 0.5101 & 6466 \\
\hline 55 & SLP 01-20 & ign riolítica & Tis & $32.5 \pm 0.8$ & 100.7813 & 22.1969 & $\mathrm{RE}$ & 54.7 & 87.4 & 5.17 & 0.6019 & 6309 \\
\hline 56 & SLP 01-06 & andesita & $\mathrm{Tcb}$ & $42.5 \pm 1.0$ & 100.7719 & 22.2258 & $\mathrm{RE}$ & 20.8 & 72.2 & 1.50 & 0.6140 & 6308 \\
\hline
\end{tabular}

SG: Sitio geológico, localización de las muestras analizadas (K-Ar), en cada complejo volcánico. Unidad geológica: estratigrafía reportada por LabartheHernández et al., (1982). $\pm 1 \sigma$; calculado siguiendo la ecuación de Cox y Dalrymple (1967), en muestras de roca más antigua a 5 Ma, y la ecuación dada por Mahood y Drake (1982) para muestras más jóvenes a 1 Ma. Fracción; RE, roca entera; m, matriz; f, feldespato; bi, biotita. \% p.: por ciento en peso de potasio. Ref. Lab.: Base de datos en el Laboratorio de Geocronología de la Universidad de Bretaña Occidental.

la andesita Casita Blanca de edad Eoceno medio. Entre el Oligoceno temprano y tardío (32-28 Ma) tuvo lugar en el área un evento volcánico voluminoso que causó principalmente el emplazamiento de derrames de lava, y en menor proporción, flujos piroclásticos. Las lavas dieron origen a cadenas de domos alineados con orientación NW-SE, cuya composición inicial fue dacítica (dacita Jacavaquero, latita Portezuelo y riodacita Zapatero). En el caso de la latita Portezuelo, se ha sugerido que la salida de la lava fue precedida por una erupción piroclástica de la ignimbrita Membrillo (Aguillón-Robles y Tristán-González, 1981; Labarthe-Hernández et al., 1982).

La última etapa eruptiva en el sur-poniente del CVA, estuvo representada por lavas riolíticas con piroclásticos asociados. Las lavas formaron domos exógenos y diques, con sílice alto y ricas en K. (riolita Cuervos, domos riolíti$\cos )$, cuya característica principal es el contenido de granate de la variedad piropo-almandino. Además de los diques riolíticos, en esta misma zona aflora un cuerpo intrusivo que se emplazo en forma de dique, nombrado como intrusivo El Negro (Labarthe-Hernández et al., 1995)

En la parte sur-oriente y oriente se encuentran remanentes de flujos piroclásticos de la ignimbrita Panalillo, que esta formada por dos miembros, el inferior son depósitos piroclásticos sin soldar, y el superior es una ignimbrita riolítica muy soldada. De acuerdo a evidencias de campo, se ha propuesto que los flujos piroclásticos de la ignimbrita Panalillo, utilizaron como conducto de salida las fallas normales principales, resultado del evento de Cuencas y Sierras (Aguillón-Robles et al., 2004; Torres-Hernández et al., 2006; Tristán-González et al., 2006). La última actividad volcánica que se sobrepuso en el Complejo Volcánico Ahualulco en el Cuaternario, fue a través de centros eruptivo puntuales, que consistió en coladas de lava basanítica (volcanes de Cúcamo y Cascarón).

\subsection{Complejo Volcánico Pinos}

Como basamento Mesozoico del Complejo Volcánico Pinos (CVP) se encuentra una secuencia vulcano-sedimentaria perteneciente al terreno tectonoestratigráfico Guerrero (Figuras $1 \mathrm{~b}$ y 3 ). Discordante, sobre el paquete anterior se depositaron sedimentos continentales correspondientes al conglomerado Zaragoza de edad Paleoceno-Eoceno (Labarthe-Hernández y Jiménez-López, 1991), similar en composición y edad a los lechos rojos de Guanajuato y Zacatecas (Edwards, 1955; Aranda-Gómez y McDowell, 1998; Aranda-Gómez et al., 2007). Sobre los sedimentos lacustres se encuentra el paquete de lavas de edad Oligoceno temprano que Aranda-Gómez et al. (2007) nombró como traquita Pinos (obteniendo una edad isotópica K-Ar de $28.7 \pm 0.9 \mathrm{Ma}$ ), y que Labarthe-Hernández y Jiménez-López (1993) nombraron como riodacita Barbecho, que es una secuencia de lavas de composición dacítica, asociada al evento de formación del domo Cerro Blanco (porción NW de la Sierra San Miguelito). En el Complejo Volcánico Pinos, la riodacita Barbecho que originó el domo de Pinos, se determinó como domo poligenético con intercalaciones de derrames piroclásticos (Aguillón-Robles et al., 1996; Aranda-Gómez et al., 2007). En la porción sur se localiza un complejo de domos formado por derrames de lava de composición riolitíca (riolita San Miguelito), que presenta mineralización de "fase de vapor" de topacios y Sn rellenando cavidades miarolíticas. Como última etapa volcánica en el Oligoceno tardío en el CVP, se emplazaron derrames piroclásticos pertenecientes a riolita Panalillo, que fueron determinadas como ignimbritas de grado alto y con características reomórficas (Torres-Hernández et al., 2003; Torres-Hernández et al., 2006; Rocha-Echavarría et al., 2006), las cuales se emplazaron a través de fallas y 


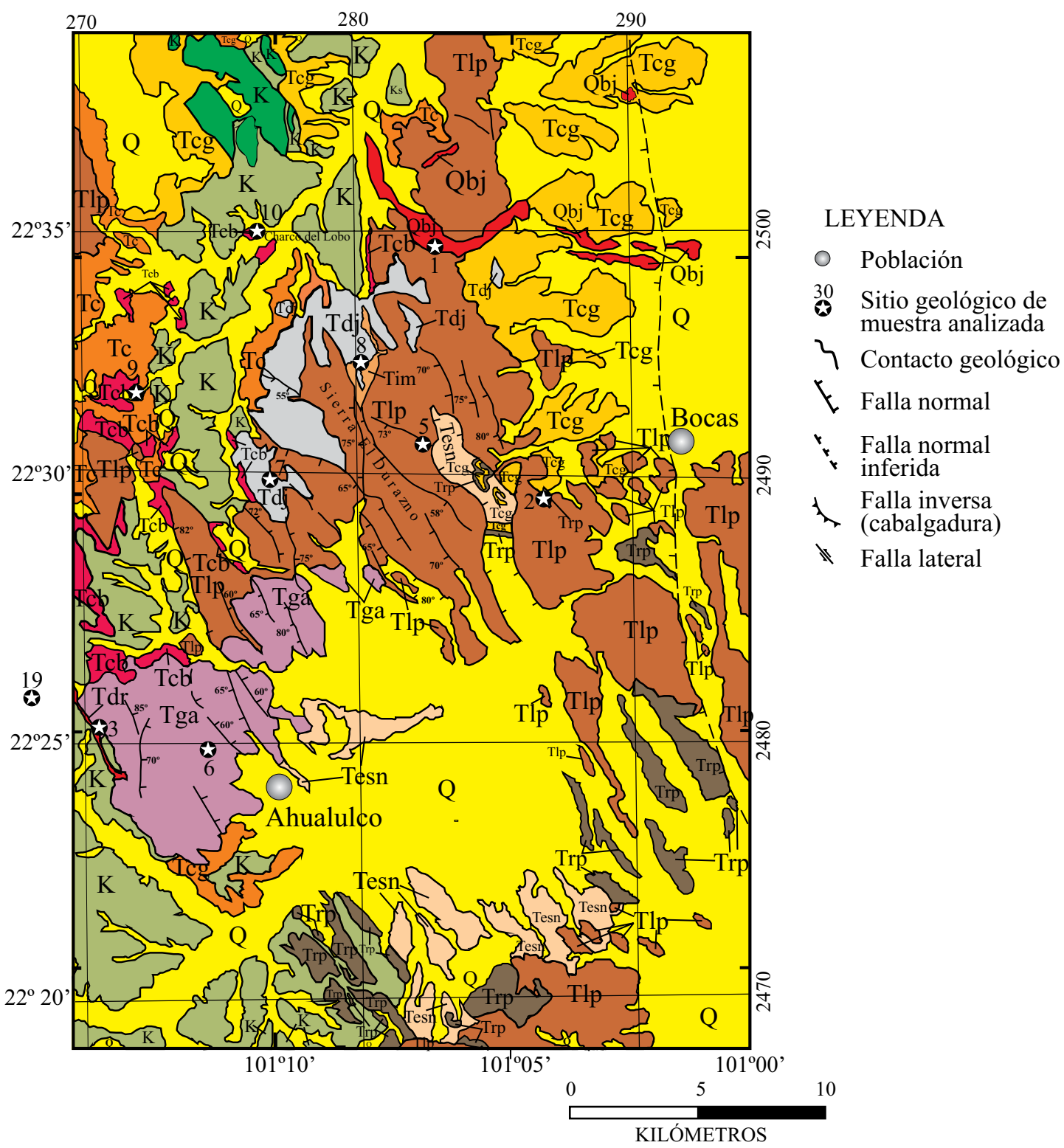

Figura 2. Mapa geológico simplificado del Complejo Volcánico Ahualulco, las unidades geológicas están referidas en la Figura 1 (mapa modificado de Labarthe-Hernández et al., 1982).

fisuras con orientación NW-SE (Torres-Hernández et al., 2006).

\subsection{Complejo Volcánico Villa Hidalgo}

El Complejo Volcánico de Villa Hidalgo (CVVH), se ubica en la porción nor-oriental del CVSLP (Figuras. 1c y 4), las unidades volcánicas descansan sobre rocas del Cretácico superior depositadas sobre el talud occidental de la Plataforma calcárea Valles San Luis Potosí, muy cerca del límite con la Cuenca Mesozoica del Centro de México (Carrillo-Bravo, 1982; López-Doncel, 2003), El CVVH lo forman domos y derrames de lava de composición dacítica-andesítica y piroclásticos de composición riolítica emplazados durante el Oligoceno. Las lavas dacíticas formaron dos domos principales en las inmediaciones del poblado de Villa Hidalgo (dacita Jacavaquero); posteriormente se encuentran depósitos piroclásticos (ignimbrita Santa María), que están intercaladas entre dos flujos de lavas andesíticas correspondientes a la andesita Villa Hidalgo y San Lorenzo (Figuras. 1 y 4). Hacia su parte oriental y sur occidental se localizan aparatos volcánicos por donde salieron lavas basaníticas durante el Cuaternario, entre ellos el maar de la Joya Honda (Aranda-Gómez y Labarthe-Hernández, 1977; LabartheHernández, 1978; Labarthe-Hernández et al., 1982), que se asocia a derrames de lavas máficas y contiene xenolitos del manto y de la base de la corteza (Aranda-Gómez et al., 1993). 


\subsection{Complejo Volcánico La Repartición}

El Complejo Volcánico La Repartición (CVR), se ubica al norte del CVVH y en la porción centro-norte del CVSLP (Figuras. 1d y 5), al poniente del ejido Llanos del Carmen. Fue descrito recientemente por Barboza-Gudiño et al. (2001). Sobre este complejo, solo se habían reportado con anterioridad la presencia de algunos derrames de basalto, cuando se encuentran en realidad depósitos de flujos piroclásticos correspondientes a la ignimbrita Santa María (Tis), un conjunto de lavas dacíticas ricas en potasio (Tdj) y una secuencia de flujos de andesitas a basaltos (Tar), emplazados a lo largo de fallas de orientación NW. En estrecha relación con estos derrames lávicos y las fallas mencionadas se observa un patrón de diques tuficíticos y acumulaciones de material piroclástico y epiclásticos. Esta sucesión volcánica se emplazó en el Oligoceno-Mioceno y descansa discordantemente sobre calizas arrecífales cretácicas de la Formación El Abra de la Plataforma Valles-San Luis Potosí (Carrillo-Bravo, 1971; Barboza-Gudiño et al., 2001).

\subsection{Complejo Volcánico Sierra San Miguelito.}

El Complejo Volcánico Sierra San Miguelito (CVSSM) se localiza en las inmediaciones sur y occidente de la ciudad de San Luis Potosí, y su rasgo morfológico más conspicuo lo constituye la Sierra San Miguelito (Figuras. 1e y 6). En este complejo los productos volcánicos son de edad Oligoceno medio y Mioceno. La secuencia oligocénica (31 a $26 \mathrm{Ma}$ ) está formada por lavas e ignimbritas de

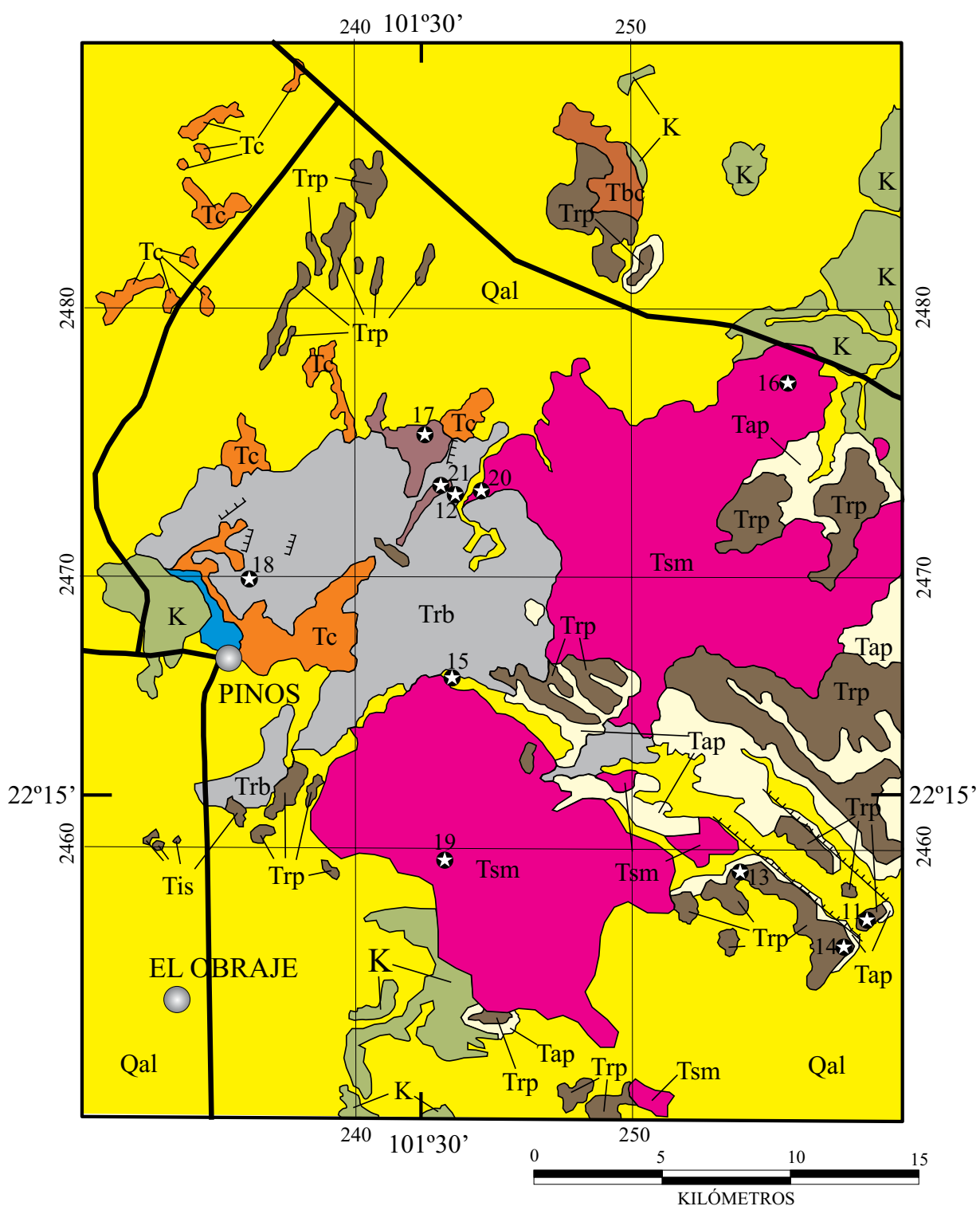

Figura 3. Mapa geológico simplificado del Complejo Volcánico Pinos (mapa modificado de Aguillón-Robles, 1983). Columna geológica en Figura 1. Leyenda del mapa ver Figura 2. 
distribución amplia. Al inicio de la actividad volcánica del CVSSM está formada por derrames de lava de composición riolítica (latita Portezuelo y riolita San Miguelito) que formaron domos exógenos. Solo de manera aislada y restringida se observan depósitos piroclásticos separando los flujos de lava que forman los domos más grandes (Cerro Grande y Cerro El Potosí). La serie de domos se emplazaron siguiendo estructuras regionales de orientación NWSE (Labarthe-Hernández y Jiménez-López, 1992; 1993; 1994); Tristán-González 1986; Aguillón-Robles et al. 1994; Rodríguez-Ríos 1997) y algunos de ellos, sobre todo los de la porción poniente de este complejo, siguieron un patrón semicircular (Torres-Hernández et al., 2008).

Gran parte del volumen de rocas volcánicas del CVSSM lo forman los domos riolíticos San Miguelito y El Zapote, entre las cuales se encuentra intercalado un paquete grueso de depósitos de flujos piroclásticos pertenecientes a la ignimbrita Cantera y San José con una edad $29 \mathrm{Ma}$, que se extienden en la mayor parte de la Sierra San Miguelito.

Entre los 27 y $28 \mathrm{Ma}$, hubo actividad volcánica puntual de composición básica siguiendo las fallas principales que seccionan la Sierra San Miguelito de orientación NW-SE (basalto La Placa) e ignimbritas de alto grado y flujos sin soldar de la riolita Panalillo, la cual sugiere una actividad bimodal ocurrida principalmente al poniente del CVSSM dentro de la fosa de Bledos.

Toda la secuencia de la Sierra San Miguelito se encuentra afectada por una gran cantidad de fallas normales con ángulo alto, que se acomodaron en patrón en dominó y formando fosas tectónicas estrechas que bascularon los bloques al NE (Labarthe-Hernández et al., 1982; LabartheHernández y Jiménez-López, 1992; 1993, 1994; Xu et al., 2004; 2005; Torres-Hernández et al., 2006),

La última actividad volcánica del CVSSM ocurrió en el Mioceno con la emisión de lavas que varían de basálticas (basalto Cabras), traquíticas (traquita Los Castillo) y riolíticas (riolita Cerro Reyna) que fueron emitidas en un periodo de tiempo muy breve entre los 21.5 y $20.3 \mathrm{Ma}$. Toda la secuencia y su ubicación temporal están esquematizadas en la Figura 1.

\subsection{Complejo Volcánico Santa María}

El Complejo Volcánico (CVSM), se localiza a unos 30 km al sur de la ciudad de San Luis Potosí (Figuras. 1f y 7); está limitado en su porción NW por la fosa tectónica conocida como Graben de Villa de Reyes (Labarthe-Hernández et al., 1982; Tristán-González, 1986), y se considera como un complejo volcánico perteneciente a la parte occidental del Campo Volcánico Río Santa María. Este complejo lo caracteriza una serie de cadenas de domos de la traquita Ojo Caliente. Su formación se inició con la emisión de coladas de lava de composición andesítica y depósitos piroclásticos riolíticos, que fueron cubiertos por un paquete de lavas con composición que varía de traquitas hasta riolita

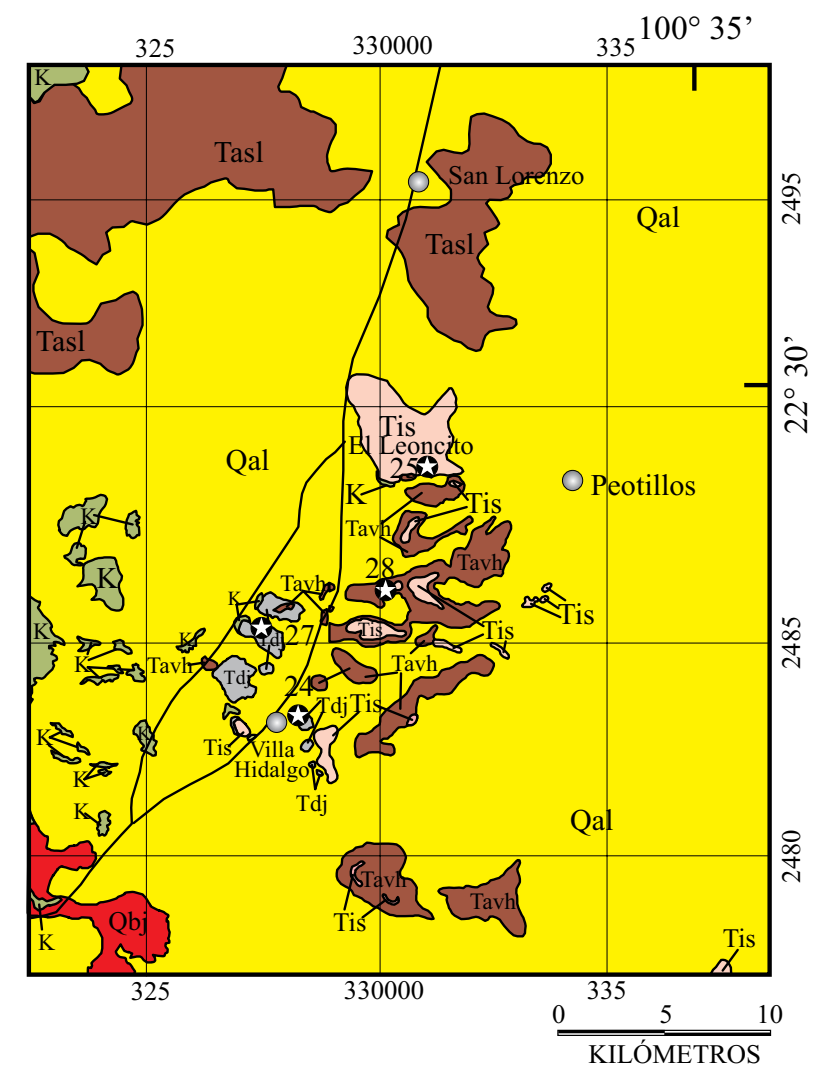

Figura 4. Mapa geológico simplificado del Complejo Volcánico Villa Hidalgo, ubicado en la porción norte del CVSLP (Modificado de Barboza-Gudiño et al., 2002). Columna geológica en Figura 1. Leyenda del mapa ver Figura 2.

de sílice alto, e intercalación esporádica en sitios puntuales con lava de composición andesita-basalto. El paquete de rocas volcánicas que forman el CVSM, descansa en su porción occidental sobre sedimentos marinos de la Formación Caracol del Cretácico superior, acumulados en la Cuenca Mesozoica del Centro de México (Carrillo-Bravo, 1982) y en su parte oriental, sobre sedimentos marinos de las facies Abra y Tamabra de la Plataforma Valles San Luis Potosí (Carrillo-Bravo, 1971; López-Doncel 2003). También esporádicamente, las rocas del CVSM se encuentran cubriendo a sedimentos clásticos continentales de la Formación Cenicera del Paleoceno-Eoceno (Labarthe-Hernández y Tristán-González, 1980, Labarthe-Hernández et al., 1982; Aguillón-Robles et al., 2004). La ignimbrita Santa María del Oligoceno temprano es la unidad basal más constante que cubre todo el CVSM, la cual es un depósito de flujo de ceniza soldado de composición riolítica, sobre el cual se emplazaron una serie de lavas que formaron domos exógenos de composición traquítica y riodacítica de la traquita Ojo Caliente y latita Portezuelo respectivamente. Entre la traquita Ojo Caliente y la latita Portezuelo, se llega a intercalar en sitios aislados la andesita Estanco. El paquete volcánico del CVSM fue afectado por una serie de fallas normales NW-SE formando un arreglo en dominó y fosas tectónicas estrechas. Algunas de estas fallas sirvieron 
como conducto para la salida de productos piroclásticos de la riolita Panalillo inferior (Tristán-González et al., 2006). El último evento efusivo del CVSM, corresponde a coladas del basalto Cabras. Esta lava basáltica se inyectó puntualmente a lo largo de las fallas normales, sobre todo en la zona de fallas marginales del hombro SE del Graben de Villa de Reyes (Tristán-González, 1986).

\section{Geocronología de los complejos volcánicos}

Se realizaron alrededor de 50 análisis isotópicos de K$\mathrm{Ar}$, en roca entera o en fase mineral, de las unidades volcánicas que conforman el CVSLP, para determinar la edad de los eventos geológicos asociados a la formación de los diferentes complejos que conforman este campo volcánico (Figura 8).

\subsection{Complejo Volcánico Ahualulco.}

Con base en evidencias estratigráficas la andesita Casita Blanca (Tcb), representa el inicio de la actividad magmática en el CVA (Figura 2). Consiste de una roca color gris oscuro con tonos verdosos, porfirítica, con un $5 \%$ de fenocristales de plagioclasa y biotita, en matriz parcialmente desvitrificada. La edad isotópica de la andesita Casita Blanca es 45.5 $\pm 1.1 \mathrm{Ma}$, obtenida con una muestra colectada en la localidad de Charco del Lobo (Tabla 1; SLP 01-31). En el arroyo Casita Blanca, se presenta como una roca de color oscuro, con $2 \%$ de fenocristales de plagioclasa de 1-2 mm, en matriz parcialmente vítrea; pertenece a una secuencia de flujos donde se distinguen horizontes de escoria (espater), piroclásticos y horizontes brechosos. . En esta localidad se obtuvo una edad de 44.4 $\pm 1.0 \mathrm{Ma}$ (SLP 01-33).

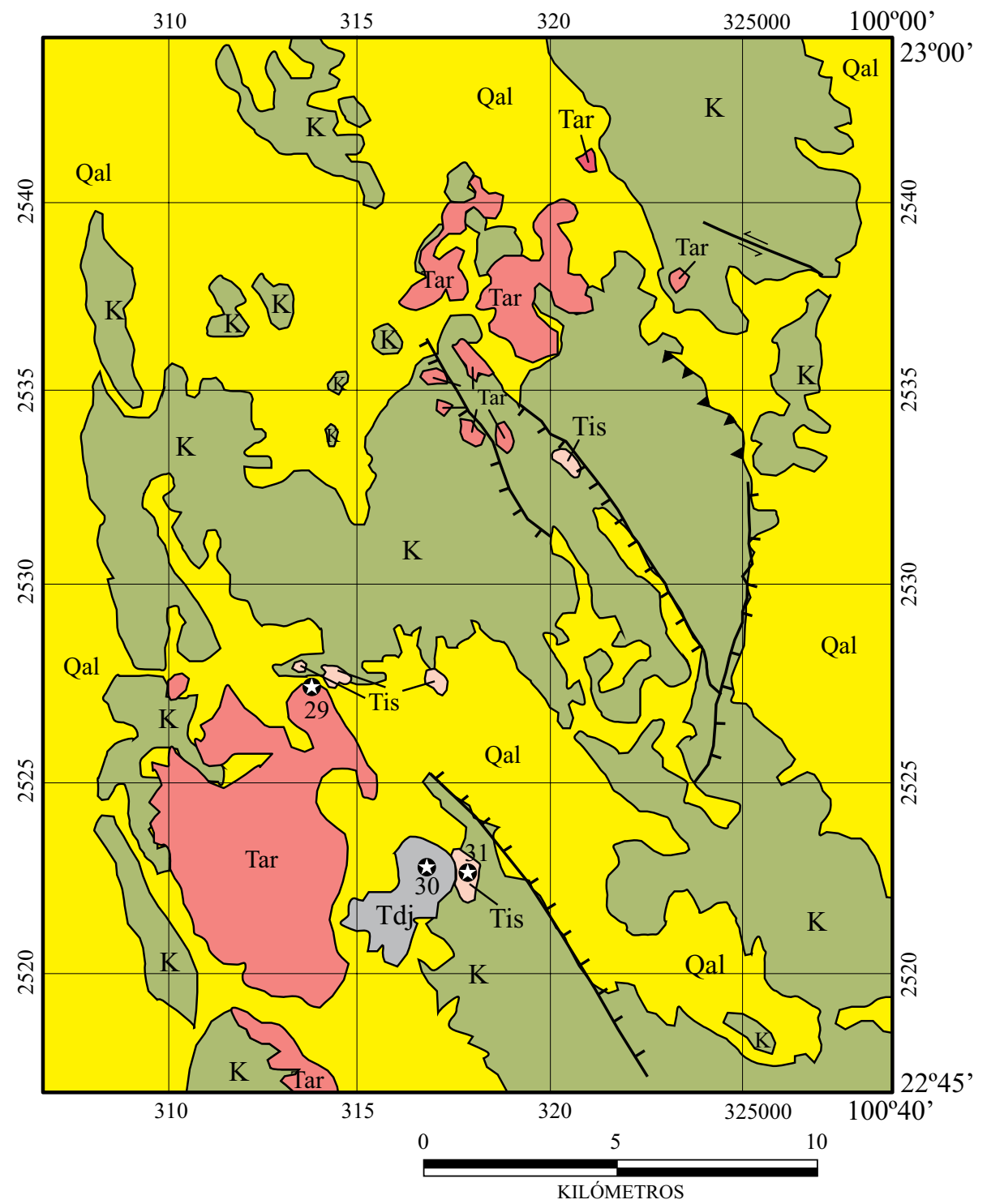

Figura 5. Mapa geológico simplificado del Complejo Volcánico La Repartición, localizado en la porción NNE del CVSLP (modificado de Barboza-Gudiño et al., 2001). Columna geológica en Figura 1. Leyenda del mapa ver Figura 2. 


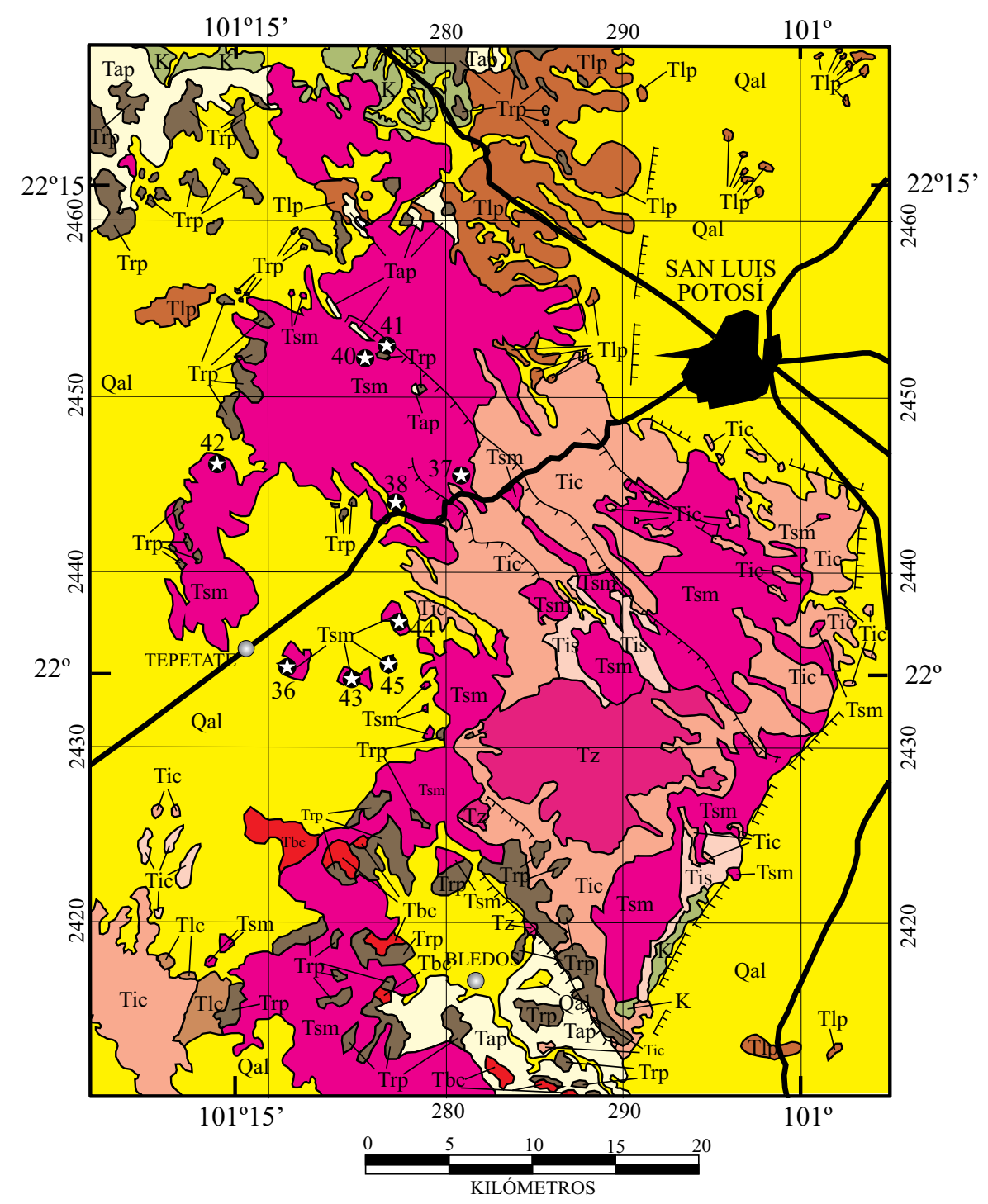

Figura 6. Mapa geológico simplificado del Complejo Volcánico Sierra San Miguelito, (modificado de Labarthe-Hernández et al., 1982). Columna geológica en Figura 1. Leyenda del mapa ver Figura 2.

Los domos de la dacita Jacavaquero (Tdj); consisten de lava de color gris claro, con $10 \%$ de fenocristales de 2-4 $\mathrm{mm}$ de sanidino, plagioclasa, hornblenda y biotita oxidada, en una matriz desvitrificada con foliación de flujo marcada. La muestra se colectó a unos $12 \mathrm{~km}$ al N de la población de Ahualulco y dio una edad isotópica de 31.6 $\pm 0.8 \mathrm{Ma}$ (Tabla 1, SLP 01-21). Después de la salida de la dacita Jacavaquero se dio la actividad piroclástica de la ignimbrita Membrillo, que se ha considerado como la base de la latita Portezuelo (Aguillón-Robles y TristánGonzález, 1981; Pallares-Ramos, 2001). La base de la ignimbrita Membrillo está sin soldar y su cima bien soldada. La parte soldada es una roca color café pardoligeramente rosa, con $10 \%$ de fenocristales de $1-2 \mathrm{~mm}$ de cuarzo y sanidino, líticos esporádicos y pómez colapsada en una matriz desvitrificada. La muestra para fechamiento de esta ignimbrita se colectó en la Sierra El Durazno, obteniéndose una edad isotópica de $32.0 \pm 0.8 \mathrm{Ma}$ (Tabla 1; SLP 01-30). La latita Portezuelo (Tlp); que es una roca color gris claro con un $15 \%$ de fenocristales de $2-9 \mathrm{~mm}$ de plagioclasa, sanidino y cuarzo aislado, con magnetita diseminada en la matriz, la cual está desvitrificada y tiene foliación de flujo. La edad isotópica de la muestra colectada en la Sierra El Durazno fue de $31.0 \pm 0.7 \mathrm{Ma}$ (SLP 0122). En la porción central del CVA, la actividad volcánica inició con la expulsión de flujo de lava de la riodacita Zapatero (Trz), la cual es una roca color café-gris, con un $30 \%$ de fenocristales de $2-5 \mathrm{~mm}$ de sanidino y plagioclasa, cuarzo subordinado, granate aislado y ferromagnesianos alterados, en una matriz desvitrificada, con numerosos micro a mesopliegues de flujo. La muestra analizada se colectó a unos $2.5 \mathrm{~km}$ al poniente del poblado de Ahualulco, la edad isotópica obtenida es de $31.2 \pm 0.7 \mathrm{Ma}$ (SLP 01-29). La actividad volcánica en la porción central 
del CVA, terminó con la erupción de lavas que formaron domos pequeños y diques (Tdr) de composición riolítica con granate. La muestra analizada se colectó a unos $8 \mathrm{~km}$ al SW de la población de Ahualulco, quedando un poco fuera del área de la Figura 2, su edad isotópica obtenida es de $31.0 \pm 0.7 \mathrm{Ma}$ (SLP 01-32). El dique El Negro, ubicado a $7 \mathrm{~km}$ al poniente de Ahualulco; es una roca color gris verdoso, porfirítica, con $15 \%$ de fenocristales de 2-15 $\mathrm{mm}$ de plagioclasa parcialmente alterada a sericita, sanidino, cuarzo y hornblenda. La edad isotópica para esta roca intrusiva fue de $29.3 \pm 0.7 \mathrm{Ma}$ (SLP 01-28). En el oriente del CVA, se llevó a cabo erupciones puntuales de los dos miembros de la ignimbrita Panalillo. El miembro Inferior es un deposito piroclástico sin soldar de oleadas piroclásticas y flujos de cáida El miembro Superior es una roca color café claro, con 5\% de fenocristales de 2-3 mm de cuarzo y sanidino, pómez colapsada y pobre en líticos, en matriz desvitrificada y bien soldada. La muestra fue colectada a $6 \mathrm{~km}$ al SW de la población de Bocas, en la porción oriental del CVA, dio una edad isotópica de $25.4 \pm$ 0.6 Ma (Tabla 1, SLP 01-24).

Traslapado sobre el CVA, hubo un evento eruptivo ocurrido en el Cuaternario que se dio de manera puntual, el cual se restringe a dos volcanes Cúcamo y Cascarón. El basalto Cúcamo se correlaciona con el basalto Las Joyas (Qbj; Labarthe-Hernández, 1978; Aranda-Gómez et al, 1993), es una roca color negro, afanítica, con fenocristales aislados de olivino. Contienen nódulos pequeños de lherzolita, la muestra analizada se recolectó en la margen sur del cráter del volcán de Cúcamo; obteniéndose una edad isotópica de $1.5 \pm 0.8 \mathrm{Ma}$ (Tabla 1, SLP 25-25). Para el volcán de Cascarón, Idier (2003), reportó una edad isotópica en roca entera de, $1.23 \pm 0.10 \mathrm{Ma}$ (coordenadas UTM, 291 701-2488 060).

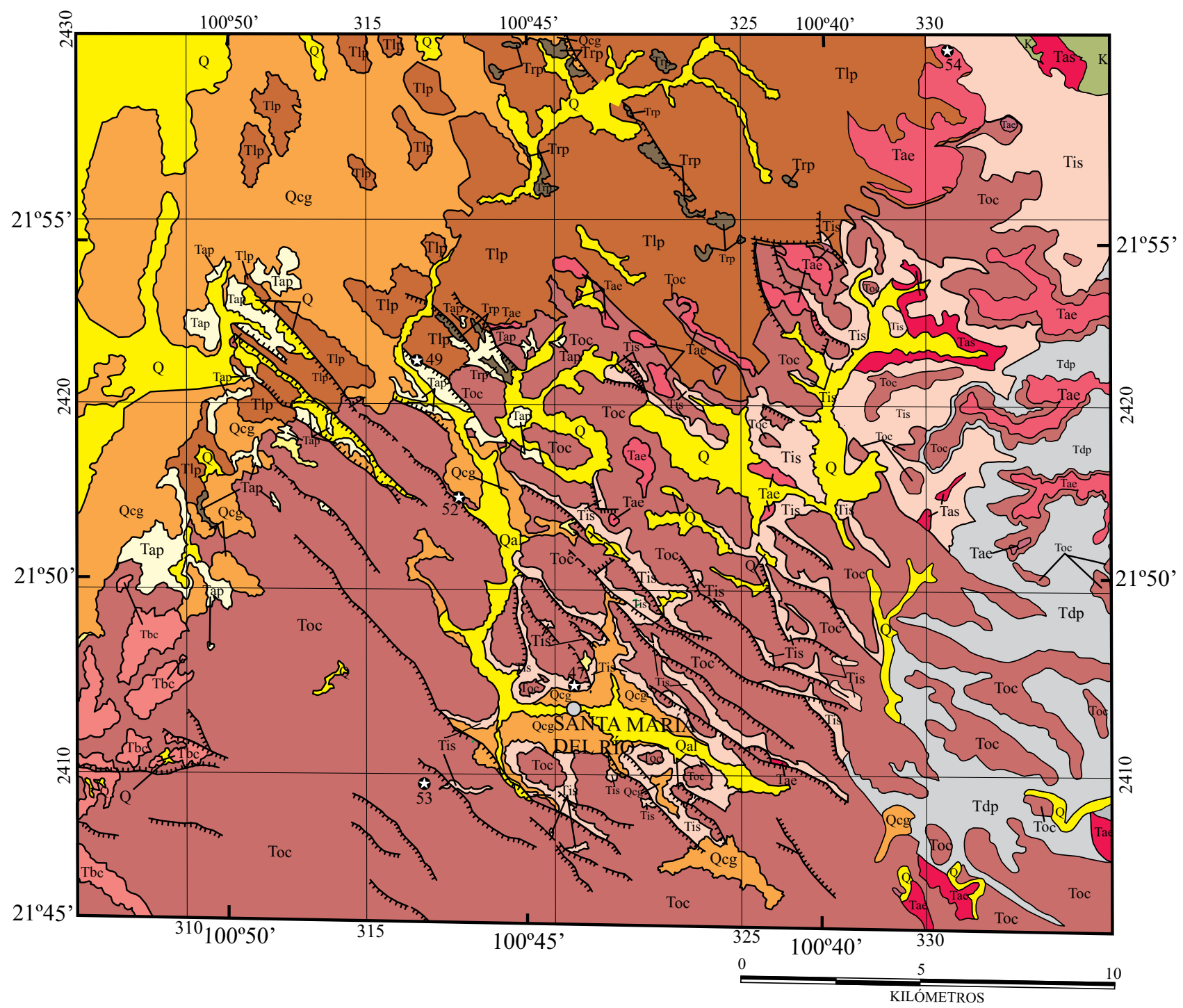

Figura 7. Mapa geológico simplificado del Complejo Volcánico Santa María (CVSM), ubicado hacia el SSE del CVSLP (modificado de Aguillón-Robles et al., 2004). Columna geológica en Figura 1. Leyenda del mapa ver Figura 2. 


\subsection{Complejo Volcánico Pinos.}

En el CVP, se emplazaron un complejo de domos de composición intermedia (traquítico), que se han nombrado como dacita Barbechos y domos de composición riolítica, finalizando con derrames piroclásticos que dieron origen a una ignimbrita de grado intermedio a alto (AguillónRobles et al., 1996; Torres-Hernández et al., 2006; RochaEchavarría et al., 2006). El domo traquítico de la dacita Barbechos está conformado por rocas que varían de 25 a $30 \%$ de fenocristales con una fase mineralógica de sanid ino $>$ plagioclasa $>>$ hiperstena $>>$ minerales opacos $>>$ apatito + zircón. Además, se ha considerado que estas rocas son producto de mezcla de magmas (Aranda-Gómez et al., 2007). El evento de formación del domo se inició desde los 31.1 Ma, finalizando alrededor de los 28.6 Ma (Tabla 1; SLP 01-03, SLP01-08), que son congruentes a las edades reportadas para las rocas traquíticas del domo de Pinos (Aranda-Gómez et al., 2007). Se ha considerado que el CVP está formado de cuando menos tres domos poligenéticos (Rodríguez-Ríos, 1997).

Después de formación del domo traquítico, se emplazó un complejo de domos riolíticos que se determinó como la unidad volcánica de la riolita San Miguelito (AguillónRobles et al., 1994b; Aguillón-Robles, 1983); los derrames de lavas riolíticas iniciaron su emplazamiento con un evento explosivo y posteriormente con etapas pasivas que generaron derrames de lavas de 1 a $2 \mathrm{~km}$ de longitud. Las rocas tienen 20 a $25 \%$ de fenocristales de cuarzo, sanidino y en menor proporción cristales de biotita; el tamaño de los fenocristales varían desde $0.5 \mathrm{~mm}$ hasta $<1 \mathrm{~mm}$, con foliación de flujo muy marcada que en ocasiones dan a la roca un aspecto finamente laminado. En algunos sitios estas rocas contienen cristales de topacio hasta de $5 \mathrm{~cm}$ de largo. El emplazamiento del complejo de domos se inició desde los $32.0 \pm 0.7$ (SLP 01-01), terminando alrededor de los $29.1 \pm 0.7$ (SLP 01-12).

Posteriormente, se emplazaron una serie de derrames piroclásticos, los cuales Torres-Hernández et al. (2006), los han considerado asociados a la tectónica extensiva de la región que ha originado fallas con orientación NW-SE y que han formado una estructura tipo semi-graben en la porción meridional del complejo volcánico, originándose ignimbritas de grado medio a alto (riolita Panalillo) con $5-10 \%$ de fenocristales de cuarzo, sanidino y pómez totalmente colapsada con una matriz totalmente desvitrificada; estas ignimbritas se emplazaron entre los $29.0 \pm 0.9$ y los $28.3 \pm 0.9$ Ma (Tabla 1; SLP 01-11; SLP 01-10; SLP 01-09)

\subsection{Complejo Volcánico Villa Hidalgo.}

El Complejo Volcánico de Villa Hidalgo (CVVH), la secuencia volcánica está compuesta por lavas que formaron domos, flujos piroclásticos asociados y derrames composición andesítica-dacítica con una edad isotópica obtenida en roca entera de 30.5 $\pm 0.7 \mathrm{Ma}$ (SLP 01-35), andesitas a basaltos de color oscuro y grano fino, frecuentemente con un intenso diaclasamiento, con edad isotópica de 31.6 $\pm 0.7 \mathrm{Ma}$ (SLP 03-01), y por ignimbritas riolíticas de grano medio a fino, ricas en cuarzo $(31.0 \pm 0.7 \mathrm{Ma}$; VH-153), edad isotópica obtenida en la unidad considerada como ignimbrita Santa María por Labarthe-Hernández et al. (1982).

Finalmente, como parte del mismo CVVH en su porción sur, se tiene la presencia de basaltos a basanitas del Plioceno-Pleistoceno que ocurren en forma de conos cineríticos, derrames y estructuras tipo maar o xalapazcos rodeados por anillos de tobas y brechas piroclásticas productos de explosiones freatomagmáticas. En una muestra proveniente de estos volcanes máficos se obtuvo una edad isotópica de $1.01 \pm 0.8 \mathrm{Ma}$ (TG 43).

\subsection{Complejo Volcánico La Repartición.}

El Complejo Volcánico La repartición (CVR), es un conjunto de domos de andesita color gris medio con gran cantidad de hornblenda, que fueron extravasados alrededor de los 32.1 $\pm 0.5 \mathrm{Ma}$ (Tabla 1; SLP 00-20), y una sucesión de unidades volcánicas y sedimentos continentales aluviales a lacustres intercalados. De manera general, se pueden describir como sigue: sobreyaciendo a las calizas y margas cretácicas, existe un conglomerado formado por clastos de caliza, con tamaños que van desde guijarros hasta bloques rodeados por una matriz arenosa calcárea. Este conglomerado ocasionalmente contiene rodados aislados de andesitas y dacitas, es sobreyacido por una ignimbrita bien soldada, de grano medio a grueso, rica en cuarzo, en donde se obtuvo una edad isotópica en roca entera de 32.5 $\pm 0.8 \mathrm{Ma}$ (Tabla 1; SLP 01-15). Esta ignimbrita tiene un espesor pequeño, desde $0.5 \mathrm{~m}$ hasta 5 a $10 \mathrm{~m}$, y es sobreyacida por un conglomerado poligmítico que contiene clastos de la misma ignimbrita y de andesitas de color gris claro a dacitas, siendo los principales componentes de esta roca fragmentos bien redondeados de margas de color gris a amarillo ocre. Finalmente, sobreyacen a este depósito algunos derrames intermedios a máficos color oscuro y en lugares vesiculares. Estas rocas tienen una edad isotópica de 20.7 \pm 0.5 Ma (SLP 01-13). Se observan también depósitos piroclásticos de color blanco a blanco amarillento formados principalmente por ceniza, intercalados con derrames de andesita-basalto alcanzando cada flujo y cada horizonte de material piroclástico entre 5 y $10 \mathrm{~m}$ de espesor, teniendo una sucesión de hasta 5 derrames con sus respectivos horizontes de material piroclástico alternados, como en el caso de la Mesa El Pollo, en la parte noreste de este complejo volcánico (Figura 5). Esta secuencia es a su vez atravesada por diques piroclásticos de color gris claro a rosado de grano fino y ocasionalmente más grueso hacia el centro de cada dique, su espesor varía desde unos 


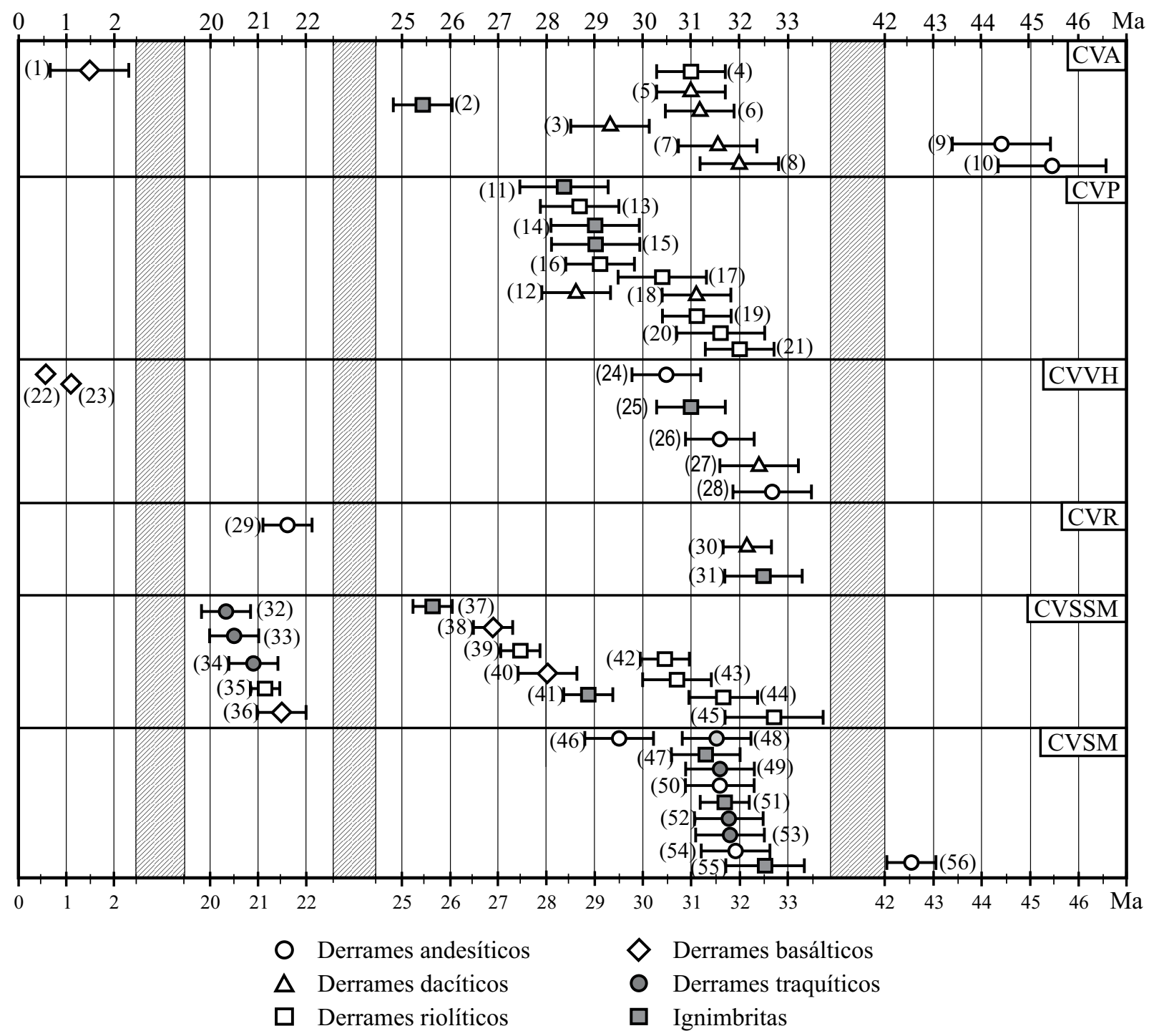

Figura 8. Diagrama que muestra las edades isotópicas, obtenidas por el método K-Ar, de muestras provenientes del CVSLP. Las muestras se clasifican de acuerdo a su litología y se establecen grupos que corresponden a los complejos volcánicos discutidos en el texto. Los números entre paréntesis, corresponden al sitio geológico (SG) de la Tabla 1. Las barras de error equivalen a $\pm 1 \sigma$; para las muestras 22 y 23 , se eliminó la barra de error por ser más pequeña que el símbolo. Clave de abreviaturas empleadas: CVA, Complejo Volcánico Ahualulco; CVP, Complejo Volcánico Pinos; CVVH, Complejo Volcánico Villa Hidalgo; CVR, Complejo Volcánico la Repartición; CVSSM, Complejo Volcánico Sierra San Miguelito; CVSM, Complejo Volcánico Santa María. Las columnas asuradas, indican ausencia de vulcanismo.

centímetros de ancho, hasta cerca de un metro los de mayor espesor.

\subsection{Complejo Volcánico Sierra San Miguelito.}

El Complejo Volcánico de la Sierra de San Miguelito (CVSSM), empezó con la emisión de las lavas de la riolita San Miguelito, formada de varios derrames episódicos, con características mineralógicas que varían de un 15 a $20 \%$ de fenocristales de cuarzo, sanidino y biotita; el tamaño de los fenocristales de las muestras de los derrames mas antiguos son entre 3-4 mm, y en las más recientes el tamaño tiende a ser $<3 \mathrm{~mm}$. El primer episodio se generó de los 32.7 a los 30.4 Ma (Tabla 1; SLP 00-17, SLP 0138, SLP 01-36, SLP 01-37) y posteriormente se encuentran eventos del vulcanismo riolítico entre el Oligoceno tardío y Mioceno a los 27.4 Ma (SLP 00-19), y 21.1 Ma (SLP 9902). Hay derrames piroclásticos de la ignimbrita Cantera e ignimbrita San José Intercalados entre la riolita San Miguelito (Torres-Hernández et al., 2008). Otro derrame piroclástico riolítico del Oligoceno tardío de la Sierra de San Miguelito es la riolita Panalillo descrita por LabartheHernández et al. (1982), la dividieron en dos miembros; el miembro Inferior es un deposito de caída y flujos piroclásticos de color crema, con fenocristales de feldespato, cuarzo, en ocasiones biotita, además de fragmentos de pómez y líticos, para estos depósitos piroclásticos se 
han reportado edades menores a $(26.8 \pm 1.3 \mathrm{Ma}$, que es la edad de la ignimbrita Panalillo Superior, en roca entera; Labarthe-Hernández et al., 1982); Torres-Hernández et al. (2008), reportaron una edad de $25.7 \pm 0.4$ Ma (en feldespato obtenido del miembro Inferior de la riolita Panalillo). El miembro Superior de la riolita Panalillo consiste de pómez y ceniza totalmente soldada; contiene de 5 a $10 \%$ de fenocristales; la fase mineral es cuarzo $>$ sanidino $>$ plagiocl asa (oligoclasa), se obtuvo una edad isotópica de $28.9 \mathrm{Ma}$ (Tabla 1, SLP 00-03), esta unidad se ha interpretado como parte de un evento de vulcanismo bimodal por su intercalación con el basalto La Placa. El basalto La Placa es una roca de color gris oscura con fenocristales de 1 a $2 \mathrm{~mm}$ de plagioclasa, olivino alterado a bitownita (Torres-Aguilera, 2005), obteniéndose una edad entre $28.0 \pm 0.6$ a $26.9 \pm 0.4$ Ma (SLP 00-02; SLP 00-14).

Los últimos eventos volcánicos del CVSSM; están representados por derrames aislados de lavas basálticas con características alcalinas denominadas por LabartheHernández et al. (1982), como basalto Cabras; que contiene olivino alterado y piroxeno y Torres-Aguilera, (2005) lo definió como traqui-andesita. Se obtuvo una edad isotópica en roca entera para el basalto Cabras de $21.5 \pm 0.5$ Ma (Tabla 1; SLP 99-02; Torres-Hernández et al., 2001). Posteriormente se encuentran derrames de lava de composición traquítica nombrada como traquita Los Castillos, conformada por derrames de color café con textura microcristalina de 5 a 10\% de plagioclasas (Labarthe-Hernández et al., 1982); esta unidad volcánica se depositó entre 20.9 a 20.3 Ma (SLP 99-04; SLP 99-01; SLP 08-03; Tabla 1; Torres-Hernández et al., 2001).

\subsection{Complejo Volcánico Santa María.}

En el Complejo Volcánico Santamaría (CVSM), la unidad volcánica basal es la andesita Salitrera, que se describe como una roca color café grisáceo, gris rojizo o gris oscuro verdoso, de textura porfirítica y pilotaxítica, con $15 \%$ de fenocristales euhedrales de $2 \mathrm{~mm}$ de plagioclasas zonadas. Los ferromagnesianos por lo general están alterados a óxidos de hierro aunque se llegan a notar cristales de piroxeno que podrían ser augita, se obtuvo una sola edad isotópica de $31.9 \pm 0.7$ Ma (Tabla 1; SLP 03-02). La ignimbrita Santa María, es una roca color gris-café de textura porfirítica, con $25-30 \%$ de fenocristales de cuarzo $>$ sanidino, con ferromagnesianos alterados a óxidos de hierro, con líticos aislados color café algunos hasta de $0.5 \mathrm{~cm}$, presenta pómez colapsada. Se obtuvieron dos edades isotópica de 32.5 \pm 0.8 y $31.3 \pm 0.7 \mathrm{Ma}$ (Tabla 1, SLP 01-20, SLP 99-05, SLP 01-44). En la porción oriental del CVSM, se emplazaron flujos de lava de composición dacítica y andesítica, correspondientes a la serie Potrerillo (Labarthe-Hernández et al., 1984; Labarthe-Hernández et al., 1989), a la fecha, no se tienen edades isotópicas de la roca de esta serie. La traquita Ojo Caliente, es una roca color gris claro a café rojizo, de textura holocristalina, inequigranular, microporfídica con 5 a 10\% de fenocristales de 1-4 mm de sanidino, plagioclasa euhedrales y ferromagnesianos alterados a óxidos de hierro, que pueden ser producto de oxidación de piroxenos y anfíboles; en dos muestras diferentes se obtuvo una edad similar de $31.8 \pm 0.7 \mathrm{Ma}$ (JAG 21-03, JAG 03-03). En la porción SE de la Hoja Salitrera (coordenadas UTM, 340 636-2413 816), fuera del área del mapa de la Figura 7, aflora el intrusivo Palo Verde, que es una roca de color gris verdoso, con contenido de $35 \%$ de fenocristales, de feldespato y plagioclasa; que se clasificó como una sienita (Labarthe-Hernández et al., 1984). La edad isotópica determinada para este cuerpo intrusivo fue de $31.5 \pm 0.7$ Ma (SLP 07-03). También en el NE de la Hoja Salitrera, fuera del área de la Figura 7, se fecharon dos muestras en la zona del volcán de Atotonilco, la primera corresponde

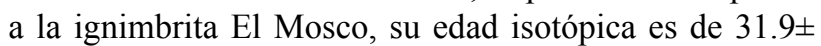
$0.7 \mathrm{Ma}$ (coordenadas UTM 352 020-2430 580) y otra que corresponde a la ignimbrita El Órgano de $31.7 \pm 0.5 \mathrm{Ma}$ (coordenadas UTM 347 050-2430 420)

En sitios aislados del CVSM, sobre las lavas de la traquita Ojo Caliente, se encuentra la andesita Estanco (Labarthe-Hernández et al., 1984;) o también nombrada Agua Fría (Aguillón-Robles et al., 2004), sus coladas son de poca longitud y espesor (Labarthe-Hernández et al., 1984); es una roca color verdoso a gris oscuro, con fenocristales de plagioclasa de menos de $2 \mathrm{~mm}$, escasos de biotita y olivino. Su textura es por lo general afanítica en matriz vítrea, parcialmente desvitrificada, con foliación de flujo. Se obtuvo una edad de $29.5 \pm 0.7 \mathrm{Ma}$ (TG 52). Sobre la traquita Ojo Caliente y remanentes de la andesita Estanco, se localizan en la porción norte y nor-poniente del CVSM, las lavas dacíticas-riodacíticas de la latita Portezuelo, las cuales forman domos exógenos de dimensiones variables (Tristán-González, 1986). La edad de la latita Portezuelo para el CVSM, no se obtuvo, sin embargo, para una muestra proveniente de su localidad tipo en el Campo Volcánico de San Luis Potosí es de $30.6 \pm 1.5$ Ma (Labarthe-Hernández et al., 1982). El basalto Cabras es una roca de color negro de textura microporfirítica y pilotaxítica, con microfenocristales de andesina y olivino alterado, la matriz es de microlitos de plagioclasa. Una muestra del basalto Cabras colectada para el análisis isotópico K-Ar en roca entera, del Cerro El Pelillo localizado muy cerca del limite poniente del área de la Figura 7 (fuera del área, coordenadas UTM, 304 968-2408 801) se obtuvo una edad de $29.5 \pm 0.7 \mathrm{Ma}$.

\section{Conclusión y Discusión}

La investigación de nuestro grupo ha llevado a identificar dentro del CVSLP, 5 complejos volcánicos, cada uno de ellos con características petrológicas y evolución muy particular, pero correlacionables entre sí. La geocronología de las distintas secuencias en cada uno de ellos, realizada por 
el método K-Ar, ubican temporalmente a la actividad magmática desde el Eoceno medio hasta el Cuaternario, señala, especialmente dos etapas paroxismales de vulcanismo una a fines del Oligoceno tardío y la otra a principios del Oligoceno temprano. Existen cuatro etapas de vulcanismo separadas por tres hiatus entre 42 a $32 \mathrm{Ma}$, de 27 a $22 \mathrm{Ma}$, y el último hiatus entre $20 \mathrm{Ma}$ a $1.8 \mathrm{Ma}$; y en esto, como en el aspecto de génesis, presenta una evolución semejante a otras regiones de la SMO. El vulcanismo del Eoceno medio (principalmente andesítico) y el del Oligoceno (principalmente riolítico), son de naturaleza sub-alcalina, y se asocian a un régimen de convergencia típico de arco volcánico continental. El vulcanismo del Mioceno (22 a $20 \mathrm{Ma}$ ) de naturaleza bimodal, varía de sub-alcalino a alcalino y refleja un evento extensional ocurrido en la parte sur de la Provincia de Cuencas y Sierras entre 24 y $20 \mathrm{Ma}$ (Aranda et al., 2000). En general, cada una de estas etapas de vulcanismo estuvo asociado a un evento de tectónica extensiva, pero solo el de edad Mioceno y del Plio-Cuaternario tienen características alcalinas, sugiriendo un régimen intraplaca (en especial este último).

Las nuevas edades isotópicas K-Ar que aquí se reportan, son coherentes con la mayoría de las edades publicadas en los trabajos de estratigrafía del Instituto de Geología de la UASLP y de otros publicados por autores diversos sobre el CVSLP (Nieto-Samaniego et al., 1996; Nieto-Samaniego et al., 2005). Con la obtención de este paquete de nuevos fechamientos se cubre casi por completo la geocronología de las distintas unidades que faltaba por fechar, y se ha podido definir la temporalidad de los distintos eventos que dieron origen a los complejos volcánicos que se están proponiendo en este trabajo; sin embargo, aún quedan otros tópicos que se irán definiendo a medida que se cuente con mas información, tanto geocronológica como geoquímica (Figura 8).

El trabajo de estratigrafía llevó a separar el paquete volcánico que aflora al SE del CVSLP, el cual tiene una columna estratigráfica diferente al campo volcánico, por lo cual se le nombró como Campo Volcánico del Río Santa María (Labarthe-Hernández et al., 1989). Ambos campos tienen como límite el Graben de Villa de Reyes; aquí se incluye la porción occidental del CVRSM, caracterizada por la abundancia de cadenas de domos exógenos de composición traquítica; y coladas de lava de andesita basáltica que corresponden al Complejo Volcánico Santa María (Labarthe-Hernández et al, 1989; Medina-Romero et al., 2005; Aguillón-Robles et al., 2006).

\section{Agradecimientos}

El presente estudio fue realizado dentro del convenio de cooperación científica entre la Universidad de Bretaña Occidental en Brest, Francia y el Instituto de Geología de la Universidad Autónoma de San Luis Potosí, México. Al Tec. Jean-Claude Phillipet y Dr. Carlos Pallares Ramos del
Laboratorio de Geocronología de la UBO, por la elaboración de los análisis $\mathrm{K}-\mathrm{Ar}$, realizadas para este trabajo. Se agradece a la D.G. Irma Mónica Pérez González por la asesoría en la elaboración de las figuras. Un agradecimiento especial a los Drs. Lucía Capra y J. Jorge Aranda-Gómez del Centro de Geociencias campus UNAM-Juriquilla, Qro., por las sugerencias y correcciones del manuscrito original, que hiciera posible mejorar el presente artículo.

\section{Referencias bibliográficas}

Aguillón-Robles, A., Tristán-González, M., 1981, Cartografía Geológica Hoja Moctezuma, San Luis Potosí: Universidad Autónoma de San Luis Potosí, Instituto de Geología y Metalurgia, Folleto Técnico 74.

Aguillón-Robles, A. 1983, Cartografía geológica de las hojas Espíritu Santo, Pinos, El Obraje y Ojuelos, Estados de San Luis Potosí, Jalisco, Guanajuato y Zacatecas: Universidad Autónoma de San Luis Potosí, Instituto de Geología y Metalurgia, Folleto Técnico 93.

Aguillón-Robles, A., 1992, Características geológicas y ambiente tectónico de un complejo de domos riolíticos del Terciario medio en la porción meridional de San Luis Potosí: Universidad Nacional Autónoma de México, Facultad de Ciencias, tesis de maestría.

Aguillón-Robles, A., Aranda Gómez, J.J. y Solorio Munguía, J.G., 1994, Geología y tectónica de un conjunto de domos riolíticos del Oligoceno medio en el sur del Estado de San Luis Potosí, México: Revista Mexicana de Ciencias Geológicas, 11, 29-42.

Aguillón-Robles, A., Aranda Gómez, J.J. y Marín-Solís, J.D., 1994a, Noticia acerca de la presencia de sedimentos continentales y volcanismo asociado (¿Eoceno?) en la Región de Pinos, Zacatecas (resumen), en Reunión Anual, Puerto Vallarta, Jal., Unión Geofísica Mexicana, GEOS 14, 80.

Aguillón-Robles, A., Aranda Gómez, J.J. y Marín- Solís, J.D., 1994b, Una localidad recién descubierta de riolitas con granate en la Mesa Central, México (resumen), en Reunión Anual, Puerto Vallarta, Jal., Unión Geofísica Mexicana, GEOS 14, 80.

Aguillón-Robles, A., Rodríguez-Ríos, R., Leroy, J.L., Aranda Gómez, J.J. y Marín-Solís, J.D., 1996, Geología y características geoquímicas del Centro Volcánico de Pinos, Zac., en Actas INAGEQ 2, 3-8.

Aguillón-Robles, A., Tristán-González, M., Torres-Hernández, J.R., Bustos-Gutiérrez, L.A., Carrizales-Aguilar, A., 2004, Carta Geológico-Minera Santa María del Río F14-C14, San Luis Potosí y Guanajuato, escala 1:50,000: Pachuca, Hidalgo, México, Consejo de Recursos Minerales, 1 mapa.

Aguillón-Robles, A., Medina-Romero, E., Tristán-González, M., TorresHernández, J.R., 2006, Evolución tectono-magmática de la porción occidental del Campo Volcánico Río Santa María, en el Estado de San Luis Potosí y Guanajuato (resumen), en Reunión Anual, Puerto Vallarta, Jal., Unión Geofísica Mexicana, GEOS 26, 164.

Aguirre-Díaz, G., Labarthe-Hernández, G., 2003. Fissure-source origin for voluminous ignimbrites of the Sierra Madre Occidental and its relationship with Basin and Range faulting: Geology, 31, 773-776.

Aranda-Gómez, J.J., Henry, C.D., Luhr, J.F., 2000. Evolución tectonomagmática post-paleocénica de la Sierra madre Occidental y de la porción meridional de la provincia tectónica de Cuencas y Sierras, México: Boletín de la Sociedad Geológica Mexicana, LIII, 59-71.

Aranda-Gómez, J.J., Labarthe-Hernández, G., 1977, Estudio geológico de la Hoja Villa Hidalgo, S.L.P.: Universidad Autónoma de San Luis Potosí, Instituto de Geología y Metalurgia, Folleto Técnico 53.

Aranda-Gómez, J.J., Luhr, J.F., Pier, J.G., 1993, Geología de los volcanes cuaternarios portadores de xenolitos del manto y de la base de la corteza en el Estado de San Luis Potosí, México: Universidad 
Nacional Autónoma de México, Boletín del Instituto de Geología $106,1-22$.

Aranda-Gómez, J.J., McDowell, F.W., 1998, Paleogene extension in the southern Basin and Range province of Mexico: Syndepositional tilting of Eocene red beds and Oligocene volcanic rocks in the Guanajuato Mining District: International Geology Review, 40(2), 116-134.

Aranda-Gómez, J.J., Molina-Garza, R., McDowell, F.W., VassalloMorales, L.F., Ortega-Rivera, M.A., Solorio-Munguía, J.G., Aguillón-Robles, A., 2007, The relationships between volcanism and extension in the Mesa Central: the case of Pinos Zacatecas, Mexico: Revista Mexicana de Ciencias Geológicas, 24, 216-233.

Barboza-Gudiño, J.R., Mata-Segura, J.L., Martínez-De la Cruz, M., 2001, Carta geológico- minera Llanos del Carmen F14-A54 San Luis Potosí, escala 1:50,000: Pachuca, Hidalgo, México, Consejo de Recursos Minerales, 1 mapa.

Barboza-Gudiño, J.R., López-Doncel, R.A., Mata-Segura, J.L., 2002, Carta Geológico-Minera Villa Hidalgo, F14-A74, San Luis Potosí, escala 1:50,000: Pachuca, Hidalgo, México, Consejo de Recursos Minerales, 1 mapa.

Bellon, H., Quoc Buü, N., Chaumont, J., Philippet, J.C., 1981, Implantation ionique d'argon dans une cible support: application au traçage isotopique de l'argon contenu dans les minéraux et les roches: C.R. Academie des Science de Paris, 292, 977-980.

Carrillo-Bravo, J, 1971, La Plataforma Valles-San Luis Potosí: Boletín de la Sociedad Mexicana de Geólogos Petroleros, 23(1), 21-46.

Carrillo-Bravo, J, 1982, Exploración Petrolera de la Cuenca Mesozoica del Centro de México: Asociación Mexicana de Geólogos Petroleros, XXXIV(1), 21-46.

Cox, A., Dalrymple, G.B., 1967, Statistical analysis of geomagnetic reversal data and the precision of potassium-argon dating: Journal of Geophysical Research, 72, 2603-2614.

Edwards, J.D., 1955, Studies of some Tertiary red conglomerates of Central Mexico: U.S. Geological Survey, Professional Paper, 264H, 153-183.

Idier, C., 2003, Caractéristiques des evènements magmatiques EocènesOligocènes et Plio-Quaternaires du champ volcanique de San Luis Potosi (Mexique): Université de Bretagne Occidentale, Mèmoire de Licence.

Labarthe-Hernández, G., 1978, Algunos xalapazcos en el Estado de San Luis Potosí: Universidad Autónoma de San Luis Potosí, Instituto de Geología y Metalurgia, Folleto Técnico 58.

Labarthe-Hernández, G., Jiménez-López, L.S., 1991, Cartografía geológica 1:50000 de las hojas Cerritos de Bernal, Santo Domingo, El Estribo y La Herradura, estado de San Luis Potosí: Universidad Autónoma de San Luis Potosí, Instituto de Geología, Folleto Técnico 113.

Labarthe-Hernández, G., Jiménez-López, L.S., 1992, Características físicas y estructura de lavas e ignimbrita riolíticas en la Sierra de San Miguelito, S.L.P.: Universidad Autónoma de San Luis Potosí, Instituto de Geología, Folleto Técnico 114.

Labarthe-Hernández, G., Jiménez-López, L.S., 1993, Geología del Domo Cerro Grande, Sierra de San Miguelito, S.L.P.: Universidad Autónoma de San Luis Potosí, Instituto de Geología, Folleto Técnico 117.

Labarthe-Hernández, G., Jiménez-López, L.S., 1994, Geología de la porción sureste de la Sierra de San Miguelito, S.L.P.: Universidad Autónoma de San Luis Potosí, Instituto de Geología, Folleto Técnico 120.

Labarthe-Hernández, G., Jiménez-López, L.S., Aranda-Gómez, J.J., 1995, Reinterpretación de la geología del Centro Volcánico de Ahualulco, SLP: Universidad Autónoma de San Luis Potosí, Instituto de Geología, Folleto Técnico 121.

Labarthe-Hernández, G., Sánchez-Pérez, M., Vázquez-Guillén, M., 1999, Mapa geológico del Volcán Atotonilco, S.L.P., México: Universidad Autónoma de San Luis Potosí, Instituto de Geología, Folleto Técnico 125.

Labarthe-Hernández, G., Tristán-González, M., 1980, Cartografía geológica Santa María del Río, S.L.P.: Universidad Autónoma de San Luis Potosí, Instituto de Geología y Metalurgia, Folleto Técnico
67,35 .

Labarthe-Hernández, G., Tristán-González, M., 1981, Cartografía Geológica Ahualulco: Universidad Autónoma de San Luis Potosí, Instituto de Geología y Metalurgia, Folleto Técnico 70, 34.

Labarthe-Hernández, G., Tristán-González, M., Aguillón-Robles, A., 1984, Cartografía geológica 1:50,000 Salitrera, S.L.P.: Universidad Autónoma de San Luis Potosí, Instituto de Geología y Metalurgia, Folleto Técnico 94.

Labarthe-Hernández, G., Tristán-González, M., Aguillón-Robles, A., Jiménez-López, L.S., Romero, A., 1989, Cartografía geológica escala 1:50,000, de las hojas El Refugio y Mineral el Realito, Estados de San Luis Potosí y Guanajuato: Universidad Autónoma de San Luis Potosí, Instituto de Geología, Folleto Técnico 112.

Labarthe-Hernández, G., Tristán-González, M., Aranda-Gómez, J.J., 1982, Revisión estratigráfica del Cenozoico de la parte central del Estado de San Luis Potosí: Universidad Autónoma de San Luis Potosí, Instituto de Geología y Metalurgia, Folleto Técnico 85.

López-Doncel, R., 2003, La Formación Tamabra del Cretácico medio en la porción central de la margen occidental de la Plataforma VallesSan Luis Potosí, centro-noreste de México: Revista Mexicana de Ciencias Geológicas, 20(1), 1-19.

Mahood, G.A., Drake, R.E., 1982, K-Ar dating young rhyolitic rocks: a case study of the Sierra La Primavera, Jalisco, Mexico: Geological Society of America Bulletin, 93, 1232-1241.

McDowell, F.W., 1977, The Sierra Madre Occidental Volcanic Province of western Mexico: Age and major element characteristics (resumen) en EOS Trans Am. Geophys. Union, 58, 1246.

McDowell, F. W., Keizer, R. P., 1977, Timing of mid-Tertiary volcanism in the Sierra Madre Occidental between Durango city and Mazatlan, Mexico: Geological Society of America Bulletin, 88, 1479-1487.

Medina-Romero, E., Aguillón-Robles, A., Tristán-González, M., Bellon, H., Cotten, J., 2005, Características geoquímicas del vulcanismo intermedio en la porción poniente del Campo Volcánico Río Santa María en la región meridional del Estado de San Luis Potosí (resumen), en XV Congreso Nacional de Geoquímica, San Luis Potosí, Actas INAGEQ, 11, 108.

Nieto-Samaniego, A. F, Alaniz-Álvarez, S. A, Camprubí í Cano, A., 2005, La Mesa Central: estratigrafía, estructura y evolución tectónica cenozoica: Boletín Sociedad Geológica Mexicana, LVII(3), 285-318.

Nieto-Samaniego, A.F., Macías-Romo, C., Alaniz-Álvarez, S. A., 1996, Nuevas edades isotópicas de la cubierta volcánica cenozoica de la parte meridional de la Mesa Central, México: Revista Mexicana de Ciencias Geológicas, 13(1), 117-122.

Nieto-Samaniego, A. F., Alaniz-Álvarez, S.A., Labarthe-Hernández, G., 1997, La deformación cenozoica poslaramídica en la parte meridional de la Mesa Central, México: Revista Mexicana de Ciencias Geológicas, 14, 13-25.

Nieto-Samaniego, A. F., Ferrari, L., Alaniz-Álvarez, S.A., LabartheHernández, G., Rosas-Elguera, J., 1999, Variation of Cenozoic extension and volcanism across the southern Sierra Madre Occidental volcanic province, Mexico: Geological Society of America Bulletin, 111, 347-363.

Pallares-Ramos, C., 2001, Estratigrafía y estructura de la rocas volcánicas al norte del Campo Volcánico de San Luis Potosí: Universidad Autónoma de San Luis Potosí, Facultad de Ingeniería, tesis de licenciatura, $78 \mathrm{p}$.

Rocha-Echavarría, N.A., Aguillón-Robles, A., Rodríguez-Ríos, R., Bellon, H., Torres-Hernández, J.R., 2006, Petrología y geoquímica de los centros volcánicos de la Sierra de Pinos Zac y su relación con el Campo Volcánico de San Luis Potosí (resumen), en Reunión Anual, Unión Geofísica Mexicana, Puerto Vallarta, Jal., GEOS, 26, 106.

Rodríguez-Ríos, R., 1997, Caractérisation du magmatisme et des minéralisations associées du dôme de Pinos et des dômes de rhyolite à topaze du Champ Volcanique de San Luis Potosi (Mexique): Université Henri Poincaré Nancy-1, France, tesis doctoral, 357 p.

Silva-Romo, G., 1993, Estudio de la estratigrafía y estructuras tectónicas de la Sierra de Salinas, Estado de San Luis Potosí y Zacatecas: 
Universidad Nacional Autónoma de México, México, tesis de maestría, $139 \mathrm{p}$.

Steiger, R.H, Jäger, E., 1977, Subcommission on geochronology: convention on the use of decay constants in geo- and cosmochronology: Earth and Planetary Science Letters, 36, 359-362.

Stewart, J.H., Roldán-Quintana, J., 1994, Map showing late Cenozoic extensional tilt patterns and associated structures in Sonora and adjacent areas, Mexico (1: 1,000000): U.S. Geological Survey, 1 mapa.

Tardy, M., Longoria, J.F., Martínez-Reyes, J., Mitre, L.M., Patiño, M., Padilla y Sánchez, R.J., Ramírez, C., 1975, Observaciones generales sobre la estructura de la Sierra Madre Oriental: La aloctonía del conjunto Cadena Alta-Altiplano Central, entre Torreón, Coahuila y San Luis Potosí, S.L.P. México: Universidad Autónoma de México, Revista Instituto de Geología, 75(1), 1-11.

Torres-Aguilera, J.M., 2005, Caracterización petrográfica y geoquímica del vulcanismo bimodal en el semigraben de Bledos, en el Campo Volcánico de San Luis Potosí: San Luis Potosí, Méx., Universidad Autónoma de San Luis Potosí, tesis de maestría, 159 p.

Torres-Hernández, J.R., Aguillón-Robles, A., Rodríguez-Ríos, R., Bellon, H., Labarthe-Hernández, G., 2008, Un patrón semianular en el emplazamiento de un conjunto de domos riolíticos en el Campo Volcánico de San Luis Potosí: Revista Mexicana de Ciencias Geológicas (en arbitraje).

Torres-Hernández, J.R., Labarthe-Hernández., G, Tristán-González, M., Aguillón-Robles, A., Siebe-Grabach, C., Macías-Vázquez, J.L., Espíndola-Castro J.M., Aranda-Gómez, J.J., 2001, Vulcanismo y extensión: La evolución de un campo volcánico en el Altiplano Central de México (resumen), en XI Congreso Nacional de Geoquímica, Actas INAGEQ, 7(1), 58.

Torres-Hernández, J.R., Labarthe-Hernández, G., Mata-Segura, J.L., Siebe-Grabach, C., Macías-Vazquez, J.L., Espíndola-Castro, J.M., 2003, La Ignimbrita Panalillo, una reoignimbrita riolítica en el Campo Volcánico de San Luis Potosí, México (resumen) en Reunión Anual Unión Geofísica Mexicana, Puerto Vallarta, Jal., GEOS, 13, 398.

Torres-Hernández, J.R., Labarthe-Hernández, G., Aguillón-Robles, A., Gómez-Anguiano, M., Mata-Segura, J.L., 2006, The pyroclastic dikes of the Tertiary San Luis Potosí volcanic field: Implications on the emplacement of Panalillo ignimbrite: Geofísica Internacional, $45,243-253$.

Tristán-González, M., 1986, Estratigrafía y tectónica del Graben de Villa de Reyes, en los estados de San Luis Potosí y Guanajuato, México: Universidad Autónoma de San Luis Potosí, Instituto de Geología, Folleto Técnico 107, 91.
Tristán-González, M., 1987, Cartografía geológica Hoja Tierra Nueva, S.L.P., Escala. 1:50,000: Universidad Autónoma de San Luis Potosí, Instituto de Geología, Folleto Técnico 107.

Tristán-González, M., Torres-Hernández, J.R., 1992, Cartografía geológica 1:50,000 de la Hoja Charcas, Estado de San Luis Potosí: Universidad Autónoma de San Luis Potosí, Instituto de Geología, Folleto Técnico 115.

Tristán-González, M., Labarthe-Hernández, G., Aguillón-Robles, A., Torres-Hernández, J.R., Aguirre-Díaz, G., 2006, Diques piroclásticos en fallas de extensión alimentadores de ignimbritas en el occidente del Campo Volcánico del Río Santa María, S.L.P (resumen), en Reunión Anual, Unión Geofísica Mexicana, Puerto Vallarta, Jal., GEOS, 26, 163.

Tristán-González, M., Labarthe-Hernández, G., Torres-Hernández, J.R., Aguirre-Díaz, G., 2008, Post-laramidic and pre-Basin and Range deformation and its implication in the Oligocenic volcanism in the eastern part of Mesa Central, Mexico: Tectonophysics (en arbitraje).

Wark, D.A., 1991, Oligocene ash flow volcanism, Northern Sierra Madre Occidental of mafic and intermediate composition magmas in rhyolite genesis: Journal of Geophysical Research, 96(B8), 13389-13411.

Wark, D.A., Kempter, K.A., McDowell, F.W., 1990, Evolution of waning subduction-related magmatism, northern Sierra Madre Occidental, Mexico: Geological Society of America Bulletin, 102, 1555-1564.

Xu, S-S., Nieto-Samaniego, A. F., Alaniz-Álvarez, S. A., 2004, Tilting mechanisms in domino faults of the Sierra de San Miguelito, central Mexico: Geologica Acta 2 (1), 189-201.

Xu, S-S., Nieto-Samaniego, A. F., Alaniz-Álvarez, S. A., 2005, Powerlaw distribution of normal fault displacement and length and estimation of extensional strain due to normal faults: A case study of the Sierra de San Miguelito, Mexico: Acta Geologica Sinica, 79 (1), 36-49.

Manuscrito recibido: Marzo 24, 2008

Manuscrito corregido recibido: Junio 10, 2008

Manuscrito aceptado: Julio 2, 2008 